\title{
An Evaluation of the Efficacy and Safety of a Protocol to Premedicate Neonates for Elective or Semi-Urgent Intubations in a Newborn Intensive Care Unit
}

Judith Dunmire Polak

West Virginia University

Follow this and additional works at: https://researchrepository.wvu.edu/etd

\section{Recommended Citation}

Polak, Judith Dunmire, "An Evaluation of the Efficacy and Safety of a Protocol to Premedicate Neonates for Elective or Semi-Urgent Intubations in a Newborn Intensive Care Unit" (2010). Graduate Theses, Dissertations, and Problem Reports. 3010.

https://researchrepository.wvu.edu/etd/3010

This Dissertation is protected by copyright and/or related rights. It has been brought to you by the The Research Repository @ WVU with permission from the rights-holder(s). You are free to use this Dissertation in any way that is permitted by the copyright and related rights legislation that applies to your use. For other uses you must obtain permission from the rights-holder(s) directly, unless additional rights are indicated by a Creative Commons license in the record and/ or on the work itself. This Dissertation has been accepted for inclusion in WVU Graduate Theses, Dissertations, and Problem Reports collection by an authorized administrator of The Research Repository @ WVU.

For more information, please contact researchrepository@mail.wvu.edu. 
An Evaluation of the Efficacy and Safety of a Protocol to Premedicate Neonates for Elective or Semi-Urgent Intubations in a Newborn Intensive Care Unit

\author{
Judith Dunmire Polak MSN, NNP-BC \\ Doctoral Research Project submitted to the \\ School of Nursing \\ at West Virginia University \\ in partial fulfillment of the requirements \\ for the degree of
}

Doctor of Nursing Practice

Laurie Badzek, RN, J.D., Chair

Susan McCrone, Ph.D.

Greg Barretto, MD.

Morgantown, West Virginia

2010

Keywords: neonate, newborn, premedication, fentanyl, intubation, pain 


\begin{abstract}
An Evaluation of the Efficacy and Safety of a Protocol to Premedicate Neonates for Elective or Semi-Urgent Intubations in a Newborn Intensive Care Unit
\end{abstract}

Judith Dunmire Polak MSN, NNP-BC

\title{
Background
}

Intubation is a painful and stressful procedure frequently performed in the NICU, yet less than $1 \%$ of US preterm infants receive premedication. The purpose of this study is to evaluate the efficacy of an intubation protocol in the NICU to add one $2 \mathrm{mcg} / \mathrm{kg}$ dose of fentanyl prior to the procedure.

\section{Objectives}

The primary outcome was to decrease the time to successful intubation. Secondary outcomes were to increase staff knowledge regarding procedural pain, to reduce the number of attempts and to decrease adverse physiologic events.

\section{Design}

Staff attended one educational session. Pre- and post-tests assessed knowledge, beliefs, and attitudes. Data pre- and post-protocol change included: time fentanyl given, time laryngoscope placed, time to successful intubation, number of attempts, and experience of intubator.

\section{Subjects}

Fifty-five infants in the NICU were evaluated during the intubation procedure (31 prior to initiation of the protocol and 24 after protocol change). There were no significant differences in birth weight, weight at intubation, gestational age, and chronologic age between groups

\section{Results}

Fifty-three staff completed an adapted version of the Pediatric Nurses' Knowledge and Attitudes Surveys pre- and post educational session. Scores were expressed as \% of correct responses. Mean scores for the posttest were significantly higher 13.81 (92\%) than the pre-test $12.6(81 \%)$ $(p<.003)$. There were no differences in mean increase in scores within groups but significant differences were seen between groups. There was no significant difference in neonatal baseline demographics. There were no differences in time to successful intubation, number of intubation attempts, or variation in physiologic variables. NNPs performed $51 \%$ of the intubations. There was a significant difference in time to intubation (326 vs. 134 seconds; $\mathrm{p}<.003$ ) and number of attempts $(2.56$ vs. $1.61 ; \mathrm{p}<.013)$ when comparing NNPs with all other providers regardless of premedication

\section{Recommendations}

The results of this study indicate that a continuing education program about neonatal pain should be developed for all caregivers in the NICU. The lack of significant difference in time to successful intubation, number of intubations, or variation in physiologic variables of the neonates might be explained by the fentanyl dose being too low. 


\section{Acknowledgements}

In an undertaking such as this, many people share in the author's time spent in the endeavor; elations and frustrations; laughter and tears; and successes and failures. I would like to take this time to thank each one of you.

- To my committee members, Dr. Susan McCrone, Laurie Badzek and Dr. Greg Barretto thank you for your guidance, time, and leadership throughout the life of this project. You were wonderful teachers. I particularly want to thank Dr. McCrone for her mentorship, for being my cheerleader at times when I could not, and sometimes knowing me better than I knew myself. I could not have completed this without you.

- To Stacey Culp - for your invaluable statistical support and patience in answering my many queries and questions.

- To my mother, Kate Dunmire - my nursing hero and role model. I am the nurse that I am because of the joy I saw in your nursing practice.

- To my father, Dan Dunmire - always a proud supporter of all my educational undertakings.

- To my husband, Mark and my daughters, Katie, Emily, and Poppy - thank you for allowing me time out of the family, your enduring patience, and quiet encouragement. I love you all. 


\section{Table of Contents}

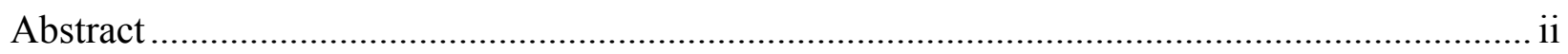

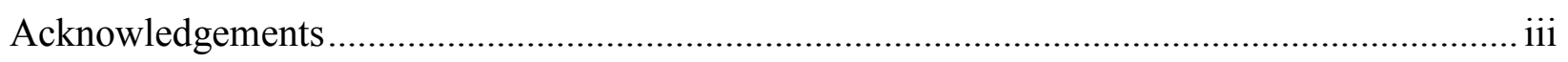

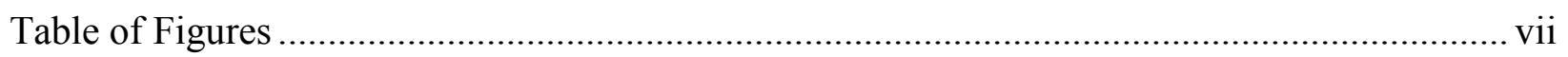

Table of Tables …................................................................................................................ viii

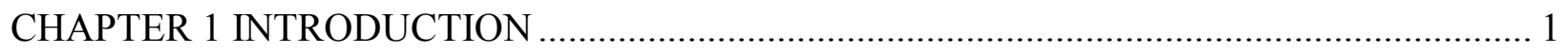

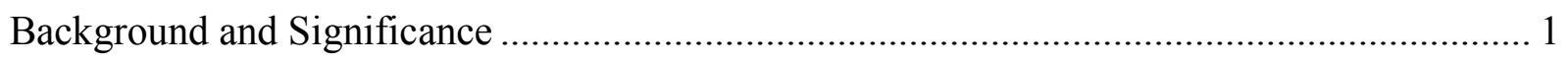

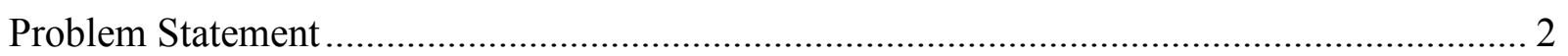

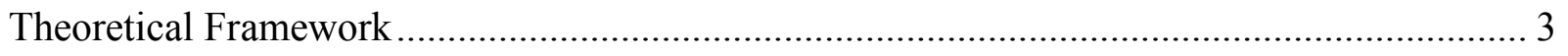

CHAPTER II REVIEW OF THE LITERATURE............................................................. 7

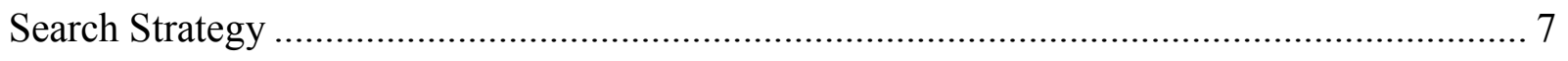

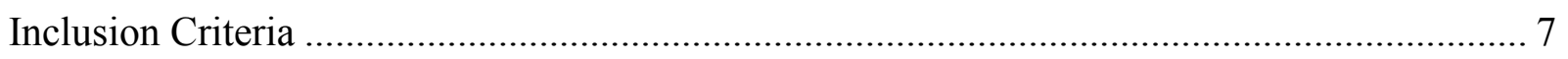

Adverse Effects of Procedural Pain in the Neonate ............................................................. 7

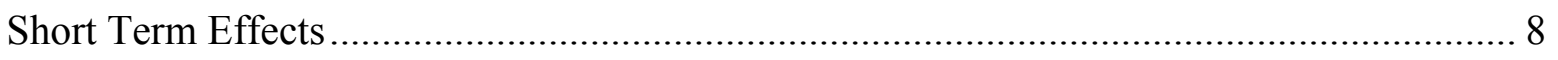

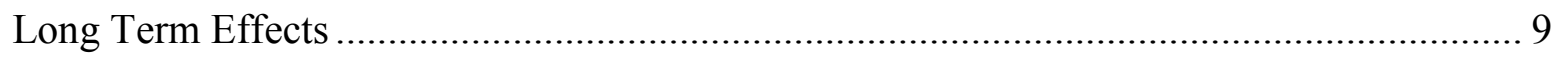

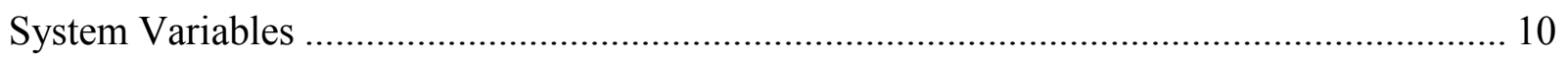

Physiologic Response to Awake Intubation .............................................................. 11

Physiologic Response to Premedicated Intubation .......................................................... 12

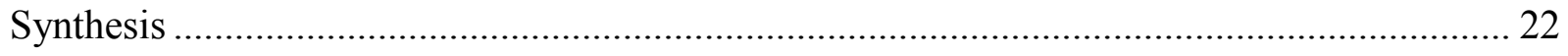

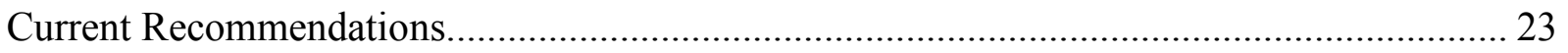


Congruence with Organizational Strategic Plan .................................................................. 24

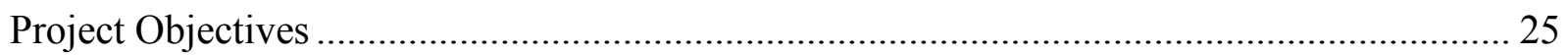

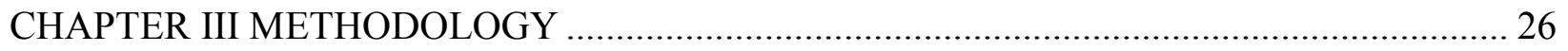

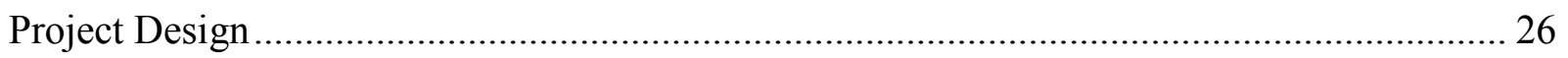

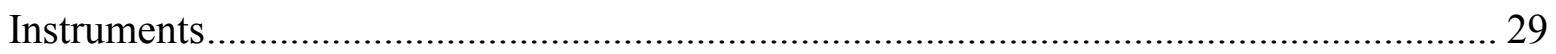

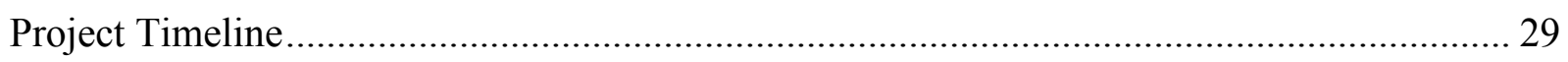

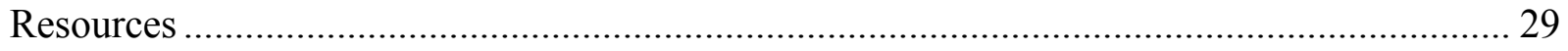

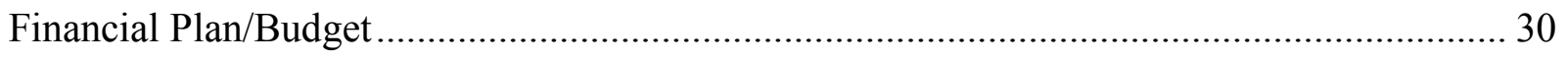

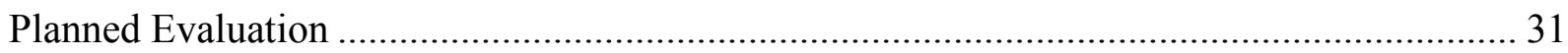

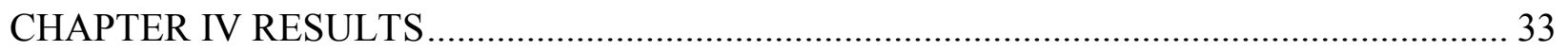

Assessment of Knowledge, Beliefs, and Attitudes of NICU Caregivers.................................. 33

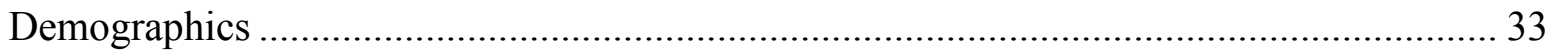

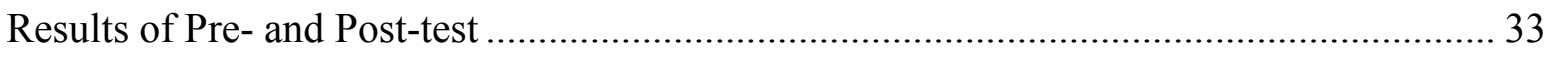

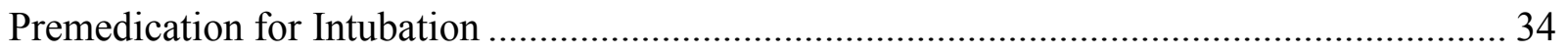

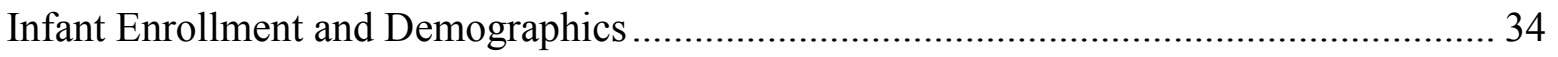

Results for Intubations Relative to Time, Attempts, and Adverse Effects ........................... 36

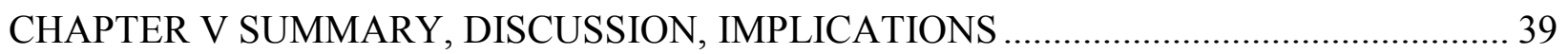

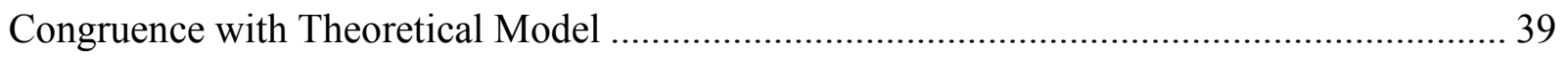

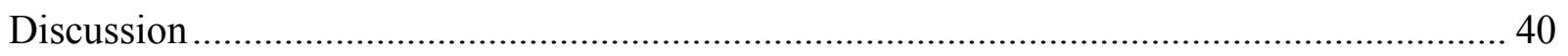

Assessment of Knowledge, Beliefs, and Attitudes of NICU Caregivers............................... 40 
Infant Enrollment and Premedication .......................................................................... 41

Results for Intubations Relative to Time, Attempts, and Adverse Effects ........................... 42

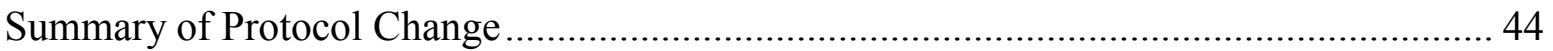

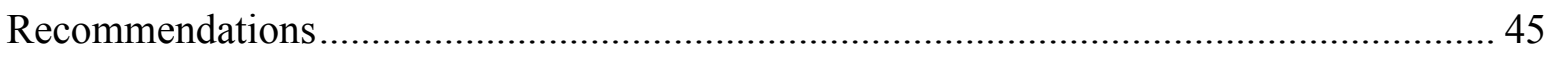

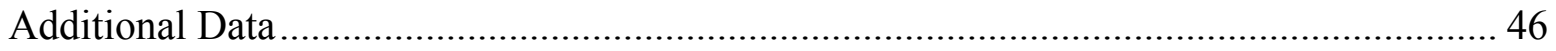

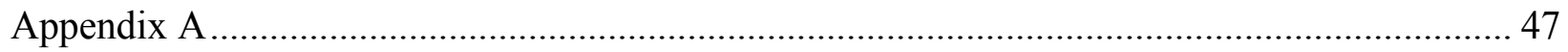

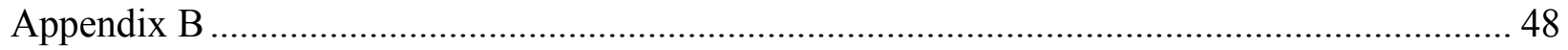

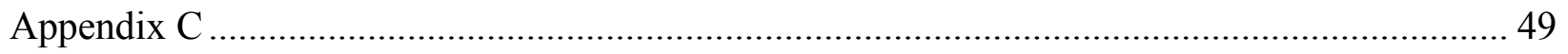

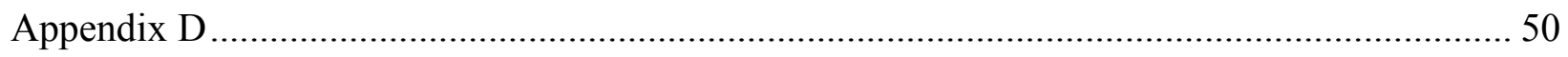

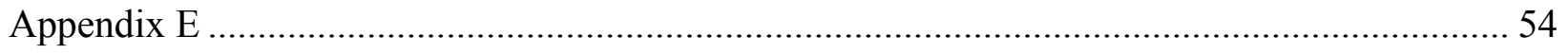

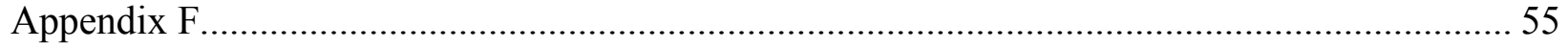

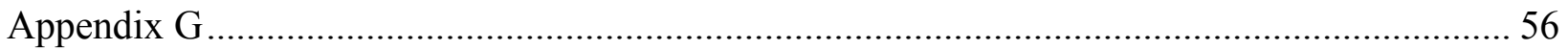

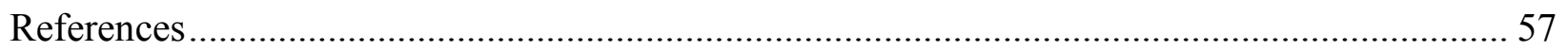




\section{Table of Figures}

Figure 1 Revised Symptoms Management Conceptual Model (Dodd et al., 2001) ..................... 4 


\section{Table of Tables}

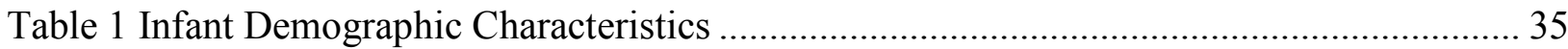

Table 2 Time to Intubation Role vs. Premedication .......................................................... 37

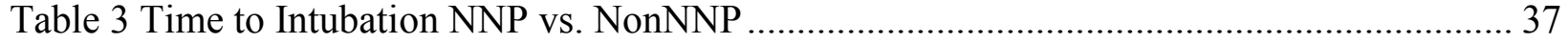

Table 4 Number of Intubation Attempts NNP vs. NonNNP ................................................ 37 


\section{CHAPTER 1 INTRODUCTION}

\section{Background and Significance}

Neonatal pain is a highly complex, dynamic, subjective experience that has been difficult to quantify and define. Before 1980, it was believed that the neonate's ability to perceive pain was blunted or nonexistent and that these neonates did not remember pain. (Anand, Phil, \& Hickey, 1987). Neonatal pain was infrequently recognized, appreciated, or treated. Since that time, the research and resources to support health care providers in neonatal pain management has expanded rapidly. It is well accepted that neonates do experience pain and stress in response to painful stimuli, and that the pain experienced by the preterm infants may actually be heightened by their nervous system immaturity (Anand, Phil, \& Hickey, 1987; Evans, 2001; Franck, Greenburg, \& Stevens, 2000). Both long-term and short-term pain responses of infants who have undergone procedures such as heelsticks and circumcision have been studied. During and shortly after these procedures, physiologic and behavioral responses have been noted. Longterm responses include an increased sensitivity to other painful procedures as well as other emotional and developmental delays (Buskila, Neumann, Zmora, Feldman, Bolotin, \& Press, 2003; Grunau et al., 2005; Lidow, 2002).

Endotracheal intubation is a traumatic and painful procedure frequently performed in the newborn intensive care unit (NICU) and delivery room (Porter, Wolf, Gold, Lotsoff, \& Miller, 1997). This procedure may cause coughing, gagging, choking, laryngospasm, hypertension, apnea, hypoxemia, bradycardia, tachycardia, and other undesirable hemodynamic disorders (American Academy of Pediatrics \& Canadian Paediatric Society, 2006; Buttner \& Finke, 2000; Kanwaljeet, 2006). Few caregivers would consider intubating an adult or a young child without 
supporting pharmacologic measures except in an emergency situation. Yet less than 1\% of preterm infants in the NICUs of the United States receive pain medication, muscle relaxants, or sedation prior to this procedure (Whyte, Birrell, Whyllie, \& Woolf, 2000). In other countries outside of the United States, such as Great Britain, France, Brazil, and Canada, a larger number of term neonates receive muscle relaxants and/or short acting analgesia prior to intubation, ranging from 8\% to 87\%. (Bonow, Piva, Garcia, \& Eckert, 2004; McKechnie \& Levene, 2007; Sakar, Schumacher, Baumgart, \& Donn, 2006; Simon, Trifa, Mokhtari, Hamza, \& Treluyer, 2004; Walter-Nicolet, Flamant, Negrea, Parat, Hubert, \& Mitanchez, 2007; Whyte, Birrell, Wyllie, \& Woolf, 2000). Premedication for the preterm infant in these international NICUs surveyed was particularly rare. A wide variety of medications were used to premedicate term infants and the evidence to support one type of medication over another was not clear.

Premedication prior to intubation has been shown to: (1) decrease the incidence of the adverse physiologic effects, (2) reduce time to successful intubation, and (3) decrease the number of attempts to intubate neonates (Bhutada et al., 2000; Shah \& Ohlsson, 2002). It is argued that the types of medications that may be used have side effects such as respiratory depression. There is little empirical evidence regarding the long-term benefits of decreasing pain and stress or providing pharmacologic support to infants during intubation, yet, ethically, pain prevention must be a priority.

\section{Problem Statement}

This capstone project evaluated the efficacy and safety of a standardized protocol for the intubation procedure in the NICU at West Virginia University Children's Hospital. After a 30 minute educational session on pain in neonates, the new protocol was introduced, which included administration of one $2-\mathrm{mcg} / \mathrm{kg}$ dose of intravenous fentanyl to all neonates prior to semi-urgent 
or elective endotracheal intubations to determine if it attenuated or ameliorated the infant's adverse physiologic responses to the procedure. In addition, the total number of attempts and the time to successful intubation were evaluated. A standardized protocol for administering analgesia prior to elective or semi-urgent intubation will provide consistency for premedication use. The purpose of this capstone project was to evaluate the efficacy of this protocol change in the NICU.

\section{Theoretical Framework}

The conceptual framework for this program of change was the Theory of Symptom Management. The Theory of Symptom Management was developed to provide a broader perspective for symptom management with a primary focus on subjective symptom experiences (Dodd et al., 2001; University of California, San Francisco School of Nursing Symptoms Management Faculty Group, 1994). This theory uses a symptomatic approach to determine intervention strategies including how and when the intervention is delivered. This approach to symptoms affects symptom experience and patient outcomes. Each of the key concepts of this theory is related to the proposed capstone project. Dodd et al, (2001) note that the symptom management model is based on six assumptions.

- The standardized clinical assessment for the study of symptoms is based on the perception of the individual experiencing the symptoms.

- Symptoms do not have to be experienced by the individual, only be at risk of symptom development to apply to this model. Intervention strategies may be initiated before symptoms are experienced. It is well documented that infants in the NICU have adverse physiologic symptoms during the intubation procedure. One dose of fentanyl was initiated in an effort to prevent these symptoms. 
- Nonverbal patients experience symptoms. The interpretation of caregivers is assumed to be accurate for purposes of intervening. Neonate's symptoms of pain are nonverbal and physiologic responses are routinely assessed during the course of the day and during procedures by the nursing staff of the NICU.

- Management strategies may be targeted at the individual, a group, a family, or the work environment. The pretreatment fentanyl intervention targeted the group of all neonates being semi-urgently intubated.

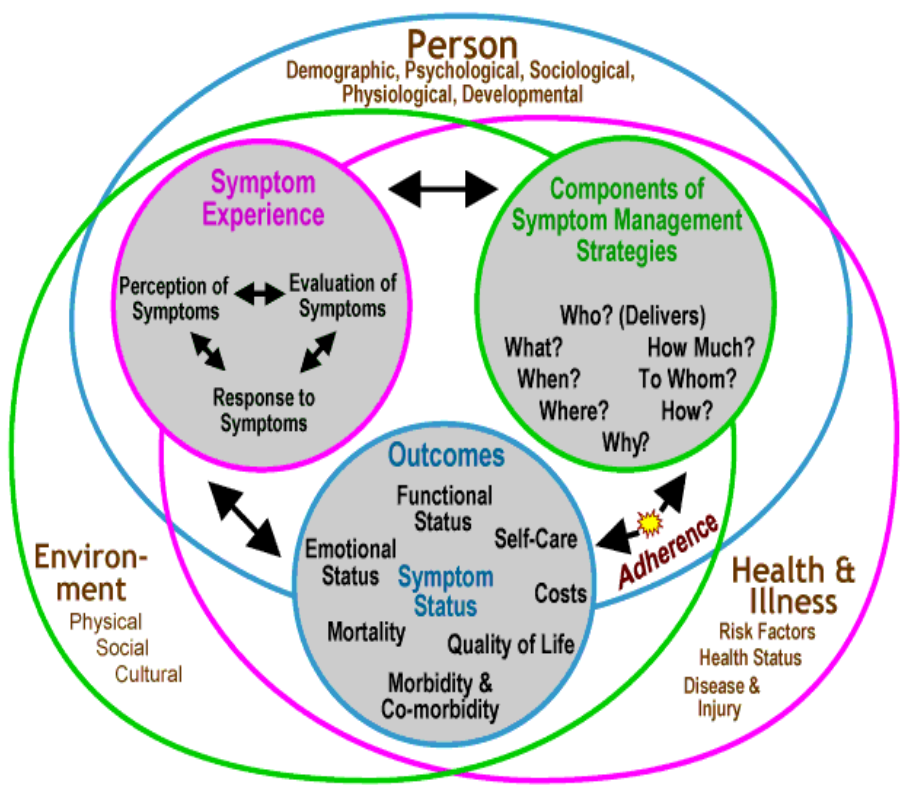

Figure 1 Revised Symptoms Management Conceptual Model (Dodd et al., 2001)

Figure 1 depicts the interrelations of the domains of nursing and the three dimensions of the model. Each is discussed in context with the capstone project.

In this model, the domains of nursing science, person, health/illness, and environment are depicted in the large ovals. These variables influence all three dimensions of the model: (a) symptom experience, (b) management strategies, and (c) outcomes.

Person variables may be individual or population-based and are affected by the 
developmental stage of the person (Dodd et al., 2001). Infants may have pain that is not treated because the symptoms are only physiologic not behaviorally based. The health and illness domain is determined by the unique variables of each individual. Obviously, a critically ill infant is more likely to require intubation than a well neonate. The gestational age of the infant and the severity of illness may exacerbate the adverse responses to the intubation procedure. The environmental domain refers to the condition or context where a symptom occurs. The intensive care setting and the volume of caregivers influence the selection of the management strategies and the outcome. Once the need for intubation is determined, the fentanyl can easily be obtained and given. Conversely, if the caregivers involved do not think that the intervention is important; they will be less likely to choose dosing with fentanyl. Staff education is an important determinant in addressing willingness to change. Review of current literature about the presence of procedural pain, including intubation, signs and symptoms, potential outcomes, and subsequent treatment of such pain were presented. Question and answer sessions for all NICU caregivers were held to assess and minimize unwillingness to change. In addition, visibility and availability of multidisciplinary change champions, across all shifts were used to promote the practice change.

The dimensions of the model are depicted in the three inner circles. The neonate perceives the symptom experience though this is evaluated and responded to by the NICU caregivers. In addition, these physiologic responses to symptoms can accentuate other negative physiologic responses such as the relationship between severe desaturations and bradycardia.

The goal of the symptom management strategy is to avoid or delay negative outcomes through intervention strategies. The nurse gives a dose of fentanyl prior to intubation to avoid the potential for adverse physiologic effects. The intervention depends on the state of the science 
for that particular symptom. At this time, there is no one medication or medication combination recommended prior to intubating neonates. Opioids are the preferred drugs of choice to manage moderate to severe pain in neonates (Anand, 2007). Morphine and fentanyl are the most commonly used opioids in the NICU. Fentanyl has a more rapid onset of action (1 to 3 minutes) and a shorter duration of action when compared with morphine (Young \& Mangum, 2007). In addition, it has minimal hemodynamic effects. It should be noted that the intervention may change over time, thus new drugs or combination of drugs may be used in the future as more research is completed.

Outcomes a re a p roduct o f s ymptom ma nagement $\mathrm{s}$ trategies a s w ell a s th e s ymptom experience. The $\mathrm{n}$ eonate $\mathrm{m}$ ay ex perience 1 ess $\mathrm{s}$ tress an $\mathrm{d} \mathrm{p}$ ain $\mathrm{w}$ hen premedicated $\mathrm{p}$ rior $\mathrm{t} o$ intubation. In addition, the duration of symptoms may be decreased as the number of attempts and the time to successful intubation drops.

In summary, the Theory for Symptom Management provides a framework for evaluating symptoms in this nonverbal population and preventing known adverse effects by proactively giving a pain medication prior to the procedure. This model, as well, provided a positive visual tool for educating NICU caregivers regarding this protocol change. 


\section{CHAPTER II REVIEW OF THE LITERATURE}

\section{Search Strategy}

To identify potential practice protocols, a thorough literature search was conducted. The terms: neonate, premedication, fentanyl, intubation, and pain were used to search the following bibliographic and abstract databases: Cumulative Index to Nursing and Allied Health Literature (CINAHL), Health Source: Nursing Academic Edition, Academic Search Premier, Biological Abstracts, and Medline (Pub Med) to identify the body of literature from 1997-2010. Language restrictions were not applied. A total of 461 abstracts were identified. The reference lists of all relevant research were also hand searched to identify other potential sources of evidence.

\section{Inclusion Criteria}

Randomized controlled trials, systematic reviews, cohort studies, and descriptive correlation studies were included. Studies in neonates that provided data on number of subjects and intubations performed were evaluated for inclusion. Other outcomes assessed for review were behavioral and physiologic variables, successful intubations, number of intubation attempts, and adverse affects. Fourteen studies were relevant and of sufficient quantity to be included. Only 12 articles could be obtained. Eight randomized controlled trials, 1 systematic review, and 2 descriptive correlation studies were reviewed. In lieu of the paucity of research available, it was decided to include the nonexperimental descriptive correlation studies because they provided relevant information.

\section{Adverse Effects of Procedural Pain in the Neonate}

The International Association for the Study of Pain (2006) and the World Health Organization (2007) define pain as an unpleasant sensory and emotional experience that stems 
from either actual or potential tissue damage or is described in terms of such damage. Pain includes not only the perception of an uncomfortable stimulus but also the response to that perception. Pain is expressed in the neonate through both physiologic and behavioral responses. As neonates are nonverbal, the assessment of neonatal pain depends on a subjective evaluation of those responses by the caregiver as well as the measurement of physiologic parameters. Both long-term and short-term pain responses of infants who have undergone procedures such as heelsticks and circumcision have been studied (Buskila et al., 2003; Franck, Greenberg, \& Stevens, 2000; Grunau, Weinberg, \&Whitfield, 2004; Peters, Koot, \& Grunau, 2003; Stevens, Johnston, \& Petryshen, 1996; Stevens et al., 2003; Stevens \& Johnston, 1994; Taddio, Katz, Ilersich, \& Koren, 1997). During and shortly after these procedures, the following physiologic and behavioral responses have been noted.

\section{Short Term Effects}

Neonatal pain produces both subjective and objective adverse effects (Peters, Koot,\& Grunau, 2003; Stevens, Johnston,\& Petryshen, 1996; Stevens et al., 2003; Stevens \& Johnston, 1994). Researchers have identified over 40 distress behaviors associated with neonatal pain, but these distress behaviors are not correlated to a specific type of pain (Shah \& Ohlsson, 2002; Warnock \& Sandrin, 2004). The subjective effects of pain are non-verbal expressions of feelings. Observable behaviors include: withdrawal from interaction, crying, moaning, irritability, grimacing, flinching, strained facial expression, or altered eye contact. Objective effects of neonatal pain are identified by bodily manifestations. Observable and concrete physiologic referents include: alterations in muscle tone, palmer diaphoresis, variations in vital signs such as tachycardia, hypertension, tachypnea, and sleep disturbances. 


\section{Long Term Effects}

The developing brain is vulnerable to environmental influences and is able to be changed by external events (Franck, Greenberg, \& Stevens, 2000). It is presumed that this vulnerability of the developing brain and pain system provide a critical window for producing long-term effects, those documented beyond the neonatal period in subsequent behavior and responses to pain and stress (Franck, Greenberg, \& Stevens). Much is still unknown about the emotional and psychological aspects of pain experienced by neonates. This does not mean that these aspects do not exist, or that they do not have harmful consequences.

The relationship between exposure to pain as a neonate and long-term perception of pain has been more clearly delineated. In a study conducted by Taddio, Katz, Ilersich, and Koren (1997), infants' age 4 to 6 months who had been circumcised as neonates showed stronger pain responses to routine immunizations than infants of the same age who had not been circumcised. Even in later life, adolescents who were premature may have stronger pain responses. Buskila et al. (2003) performed a case-control study to evaluate the tenderness thresholds in adolescents by tender-point count and dolorimetry. The preterm group was recruited through files of a tertiary referral NICU. Entry criteria included a birth weight less than $1500 \mathrm{~g}$ and gestational age less than 35 weeks. Adolescents who were born prematurely had tenderness at higher number of tender points and lower tenderness thresholds on dolorimetry. Females had more tender points and lower tenderness thresholds versus males in both the term and preterm groups.

Exposure to painful procedures in the preterm infant may increase the risk of endocrine abnormalities (Grunau, Weinberg, \& Whitfield, 2004). The investigators completed a casecontrol study assessing cortisol levels in 8-month-old (corrected chronological age), former preterm infants at baseline, after novel toy introduction, and after a developmental assessment. 
Higher cortisol levels were directly proportional to those neonates that had undergone more skin breaking procedures in the NICU. There was no relationship to gestational age at birth. The authors postulate that the exposure to pain disrupts the development of the hypothalamicpituitary-adrenal axis. They note that this disruption was not modified by treatment with morphine during the NICU stay.

\section{System Variables}

Infants in the NICU are exposed to a variety of environmental influences that can have significant negative effects on these infants. The NICU environment may have bright lights and constant noise as well as the frequent procedures potentially causing pain. This environment can have a significant negative impact on the infant (Blackburn, 1998). A preterm infant with immature organ systems is particularly vulnerable to the risk of complications from painful procedures, overexpenditure of energy, and increased stress response (Als et al., 1994). Developmentally sensitive care in the NICU may foster the growth and development of the neonate as well as support the developing systemic organization and responses (Als et al.).

Neonates are particularly vulnerable to inadequate treatment of pain, as they cannot verbalize the description or severity of their pain the way older children and adults can. They must rely on the bedside nurse's ability to correctly interpret their cues of pain, manage it appropriately, and when possible, prevent it. Discrimination between pain, agitation, and stress can be confusing, particularly for the inexperienced nurse. In addition, despite emerging scientific evidence, the attitudes and beliefs of the health care professionals involved in the care of these infants play a major role in the treatment of pain (Ellis et al., 2007). In recent years, several pain assessment tools that measure pain-related behaviors have been developed for these nonverbal term and preterm infants. The use of standardized assessment tools and the 
requirement for staff nurses' education on neonatal pain can decrease the potential for undertreatment of pain experiences in the NICU.

\section{Physiologic Response to Awake Intubation}

Awake intubation implies that the neonate will undergo direct laryngoscopy and be intubated without any prior medication. Intubating an awake adult without adequate analgesia results in intense pain. While the presence or absence of pain is more difficult to assess in the neonate, awake intubation causes a variety of adverse physiologic effects including significant changes in blood pressure, heart rate, oxygenation, and increased anterior fontanel pressure (Anand, Phil, \& Hickey, 1987; Carbajal, Eble, \& Anand, 2007; Kelly \& Finer, 1984).

Awake intubation (without preprocedural analgesia) causes both fluctuations in blood pressure and an acute hypertensive response (Bhutada, Sahni, Rastogi, \& Wung, 2000; Hassid et al. 2007; Shah \& Ohlsson, 2002). Concerns that an acute hypertensive response or systemic hypertension could potentially be harmful in the preterm neonate by increasing the risk of intraventricular hemorrhage have been raised (Shah \& Ohlsson, 2002). These changes in blood pressure can be modulated by the addition of analgesia, sedation, or anesthesia (Bhutada et al., 2000: Hassid et al., 2007; Shah \& Ohlsson, 2002)

Both bradycardia and tachycardia are reported in response to both awake and anesthetized intubation (Cook-Sather et al., 1998; Millar \& Bissonette, 1994). Bradycardia is due to increased vagal tone associated with laryngeal stimulation. Tachycardia is a direct physiologic response to pain or stress in the neonate (Shah \& Ohlsson, 2002; Warnock \& Sandrin, 2004). There is no direct evidence to suggest that neonates are compromised by these variations in heart rate during awake intubation. 
Awake intubation is associated with more severe and prolonged hypoxia than when anesthesia or analgesia is used (Lemyre, Doucette, Kalyn, Gray, \& Marrin, 2004; Shah \& Ohlsson, 2002). Preoxygenation and the use of a laryngoscope that delivers continuous oxygen can help to reduce the incidence of hypoxia during intubation (Cook-Sather et al., 1998).

Awake intubation is also associated with increased struggling and movement. As a result, several attempts at intubation are frequently necessary (Bhutada et al., 2000; Shah \& Ohlsson, 2002). Oxygen saturations are often lower because of multiple attempts to successful intubation. Successful intubation is generally faster, with fewer attempts when premedication with analgesia or a combined drug regimen is used (Dempsey, Al Hazzani, Faucher, \& Barrington, 2006: Ghanta et al., 2006; Hassad et al., 2007; Naulaers, Deloof, Vanhole, Kola, \& Devlieger, 1997; Oei, Butha, \& Lui, 2002).

Awake intubation has been associated with sustained intubation trauma. Infant movement most frequently causes this. Reducing the time to successful intubation and preventing multiple intubation attempts could potentially decrease airway damage associated with repeated intubations.

\section{Physiologic Response to Premedicated Intubation}

In a systematic review, Shah and Ohlsson (2002) found limited evidence available regarding the efficacy and effectiveness of different drugs for premedication in neonates undergoing intubation. They noted that the studies reviewed enrolled a small number of infants. Generally, the results suggested that there was an attenuation of the physiologic responses in these neonates. Premedication was also noted to shorten intubation time for the procedure. No studies addressed long-term outcomes such as neurodevelopmental outcome or heightened pain response. 
A wide variety of drugs, either alone or in combination, have been used as medications prior to intubation in elective or semi-urgent intubations in neonates, including sedatives, analgesics, and anesthetics. Although optimal drug choices have yet to be defined, these studies show interesting results evaluating advantages and disadvantages of different drug combinations.

In a randomized, placebo controlled study, Bhutada, Sahni, Rastogi, and Wung (2000) examined the effectiveness of premedication with thiopental, an anesthetic agent, on heart rate, blood pressure, and oxygen saturations during semi-elective nasotracheal intubations in 30 neonates with respiratory distress weighing greater than $2 \mathrm{~kg}$. Three infants (two in the control group and one in the treatment group) were not included in the final analysis as the data were lost during acquisition. Physiologic data was obtained through electronic monitoring with minuteby-minute measurement of heart rate, heart rate variability, mean blood pressure, and transcutaneous oxygen saturation. Baseline measurements and measurements during drug or placebo (normal saline) infusion for all variables were comparable in both groups. During intubation, heart rate and blood pressure were maintained nearer to baseline levels in the infants who received thiopental than those who received awake intubation. The control group showed a net decline in heart rate and a substantial increase in blood pressure. This trend persisted through recovery. The time to successful intubation was significantly shorter in the premedication group. This study was nonblinded, though in an attempt to limit variations in expertise and technique, only two persons performed all intubations. All data but time to successful intubation was obtained through electronic monitoring. Another limitation was the relative maturity of the cohort being treated for a variety of cardiorespiratory and surgical issues. The mean weight birth weight was $3.27 \mathrm{~kg}$. Neonates of different size and gestation and those with different diagnoses may respond differently to intubation with or without premedication. 
Dempsey, Al Hazzani, Faucher, and Barrington (2006) prospectively collected data on semi-elective intubations over a 6-month period in 33 patients who received a combination of atropine, mivacurium, and fentanyl prior to intubation. This medication combination was an established protocol in this NICU. The objective of this study was to evaluate the experience with the current medication protocol as part of an ongoing quality improvement initiative. In particular, the investigators wanted to evaluate the intubation success rate of residents with no prior intubation experience, determine the intubation conditions achieved, and evaluate the clinical effects of mivacurium in the neonate including onset and duration of paralysis and any adverse events associated with the premedication protocol. Twenty-two of the infants were intubated on the first attempt (the majority by an inexperienced intubator) and median intubation time was 60 seconds. Personnel with prior experience who attempted the primary intubation were successful on the first attempt $93 \%$ of the time. The number of desaturations less than $80 \%$ was fewer and shorter when the person performing the intubation was experienced. Muscle relaxation occurred at a mean of 54 seconds. The mean time of return to spontaneous movement was slightly less than 16 minutes. This length of paralysis could potentially require increased ventilatory support for the infant until spontaneous effort returns. Intubation conditions were scored as excellent using a validated intubation scale. Limitations of this study included the nonexperimental design and the very diverse ages and weights of the population, though the outcomes can be considered more representative of daily clinical practice in a NICU.

Ghanta, Abdel-Latif, Liu, Ravindranathan, Awad, and Oei (2006) evaluated the effectiveness and reduction of adverse effects of two premedication regimens for endotracheal intubation. The primary purpose of the study was to compare times to successful intubation and adverse hemodynamic variables between the two groups. Sixty-three infants were randomly 
assigned to receive propofol or atropine, morphine, and suxamethonium prior to nonemergent intubation. The investigators concluded that propofol was more effective than morphine, atropine, and suxamethonium as an induction agent. Sleep or muscle relaxation was achieved within one minute in both groups. Infants were intubated more than twice as fast in the propofol group. Nasal and oral trauma was less common in the propofol group. There were no differences in heart rate and blood pressure between groups. Hypoxemia was less severe in the propofol group, probably because of maintenance of spontaneous breathing. One limitation to this study was the single blinded design. Blinding of the physician was not possible as the drugs were very different in appearance, volume, and effect. In addition, the time taken to prepare 3 vials of medication would be longer than the time taken to prepare 1 vial, which may delay the procedure. Suxamethonium, a drug used commonly by anesthesiologists for rapid sequence intubation prior to surgery, is not commonly kept in the NICU. Propofol is more readily available.

In a nonblinded, randomized controlled study by Hassid et al. (2007), 33 term and preterm infants were randomized to awake intubation or sevoflurane, an inhaled anesthetic agent, as premedication before intubation. Concentrations of sevoflurane varied from $2 \%$ to $5 \%$, adjusted to achieve optimal anesthesia, measured by spontaneous breathing with loss of tonicity. Physiologic data was collected every minute and registered on a computer linked with the monitor of the infant. Intubations were considered "easier" in the sevoflurane group as measured by little to no movement and better glottic exposure. No major adverse effects (hypotension or number of desaturations) were noted in the study group compared with the control group. The incidence of bradycardia and hypertension were greater in the control group. There was no difference in duration of desaturations, but those in the control group were preoxygenated with 
$100 \%$ oxygen. The failure rate of intubation was lower in the study group, but was not statistically different. An uncontrolled mix of experience levels of those persons performing the intubations in each group could explain this difference. One limitation of this study was that the number of infants needed to treat was below that of statistical power. Though this study determined that induction of anesthesia with sevoflurane is safe and efficacious, its use in the NICU is not practical as premedication for elective or semi-urgent intubations as anesthetic agents are not routinely kept in an NICU.

Lemyre, Doucette, Kalyn, Gray, and Marrin (2004) assessed the effects of a morphine dose prior to the intubation attempt on heart rate, blood pressure, and oxygen saturations as compared with a placebo dose in 34 infants. In addition, the number of attempts to successful intubation was recorded. This double-blinded, randomized controlled study failed to demonstrate the effectiveness of morphine in reducing physiologic instability or time to performing elective intubations. Duration of hypoxemia, increases in blood pressure, and incidence of bradycardia were similar between groups. The morphine dose was given 5 minutes after randomization; peak effects are achieved in 15-30 minutes. Thus, adequate time may not have been allowed for true assessment of affect. There were several design limitations. Parents of infants who were likely to need intubation some time during their hospital stay were approached for consent after birth. Approximately half of these infants were actually enrolled and randomized. Thirteen infants never required intubation; 17 elective intubations were missed as most occurred on the night shift when no investigator was on site. The small sample size precluded elimination of a type 2 error. The investigators assumed severe hypoxemia would occur for about 30 seconds, which was not the case. They suggested that an observational study would have been useful before making this assumption. The study did not specifically address 
the disease process or the degree of illness of the infants, which could affect response to the medication. There was also significant variability in the experience of those performing the intubation. Given the small number of infants actually randomized, this might have impacted the outcomes.

Naulaers, Deloof, Vanhole, Kola, and Devlierger (1997) evaluated the effectiveness and safety of a short acting barbiturate, methohexital, prior to intubation in 18 late preterm and term infants requiring elective intubation for respiratory distress or cardiac conditions. This prospective, descriptive, correlational design assessed the effect of methohexital on sedation, relaxation, and cardiovascular effects. All infants achieved intubation in full relaxation and somnolence within 2 minutes. Most infants recovered within 5 minutes; all within 10 minutes. A slight to moderate drop in saturations occurred during intubation, particularly in those infants with heart disease. Side effects of the drug were twitching and a small drop in blood pressure. One significant limitation of this study was the diverse diagnoses of the patient group, which could potentially affect the severity of the physiologic changes measured. Disease processes included: respiratory distress syndrome with pulmonary hypertension, cyanotic congenital heart defects, chronic lung disease, and congenital defects of the lung. Though no serious side effects of the drug were noted, the range and safety of the effects of methohexital have not been studied in neonates. The investigators do not recommend regular or repeated use of this drug until further information is available on its use in the neonatal population.

The purpose of the work by Roberts, Leone, Edward, Rich, and Finer (2006) was to investigate whether using the short-acting paralytic, mivacurium, in addition to an established premedication regimen of atropine and fentanyl, would improve intubation conditions, thus decreasing the incidence and duration of hypoxia and time/number of attempts needed to 
successfully complete the intubation procedure. A total of 41 infants completed this prospective, randomized controlled trial. There were no baseline differences in demographics. The incidence and duration of hypoxemia was determined by oxygen saturation monitoring. Heart rate, blood pressure, and oxygen saturations were recorded every second. In addition, a videotape was reviewed to determine the time and number of intubation attempts and the duration of the action of mivacurium. The investigators demonstrated a reduction in time and number of attempts to successful intubation as well as a reduction in severe hypoxemia. The cumulative time of hypoxemia (saturations $<85 \%$ ) was not significantly different between groups. One limitation of the study was the inability to obtain the appropriate number of patients in a reasonable time, thus this was published as an explorative study. A total of 144 infants were required to detect a $50 \%$ difference in the primary outcome of hypoxia. An analysis of the incidence of hypoxia was completed after 30 infants were randomized. The actual difference for this outcome was $47 \%$ (55\% in the control group and $29 \%$ in the study group) compatible with the hypothesis that the addition of a paralytic to an established premedication regimen would decrease the incidence of hypoxia during the intubation procedure.

Oei, Hari, Butha, and Lui (2002) investigated the responses of 20 neonates to determine if premedication with morphine, atropine, and suxamethonium reduced the number and times of attempts by junior medical staff to achieve nasotracheal intubation as compared to awake intubation. The experimental design was a nonblinded, randomized controlled pilot trial. Blinding was not possible because of the obvious effects of muscle relaxation. Heart rate and pulse oximetry were monitored with standard bedside cardiorespiratory monitors prior to and throughout the intubation procedure. An independent staff member recorded times of beginning and completion of each intubation attempt. Lowest heart rate and oxygen saturation levels 
during each attempt were recorded. Each infant's mouth and oropharynx were examined for evidence of iatrogenic trauma. The authors concluded that the use of premedication reduced the total time and number of attempts taken to achieve successful intubation. In general, it took twice as long to intubate an awake infant. There were no statistically significant differences in intubation trauma or change in oxygen saturations, though trauma was seen more in awake infants. The lowest mean heart rate was in the control group. One limitation of this study is that it was changed to a pilot protocol after it was realized that the length of time to achieve the number of patients for statistical significance was prohibitory. In addition, although the infants in the premedication group and the awake group were statistically similar in weight, gestational age, and reasons for intubation, the age at intubation was very different (13.5 days in the control group and 1 day in the premedication group). Any infant, despite weight or gestational age, will struggle in response to a painful procedure. It is possible that younger preterm infants would struggle less than the older preterm infant. The authors suggest that stratification with larger sample sizes would be required in future studies.

In a double-blind, randomized controlled study by Silva et al. (2007), twenty preterm infants were randomized to receive either morphine and midazolam or remifentanil and midazolam prior to elective intubation to compare the intubation conditions achieved following sedation. Premedication was given 150 seconds prior to intubation. Heart rate, blood pressure, and oxygen saturations were monitored and recorded before, during, and 10 minutes following the intubation procedure. A single pediatric anesthesiologist, who was blinded, completed all intubations. Intubation conditions were scored using a four-point scale assessing ease of laryngoscopy, position of vocal cords, jaw relaxation, and movement of the extremities. In addition, pain and stress were measured using the Neonatal Infant Pain Scale (Lawrence et al., 
1993 ) and the Comfort Score (Ambuel, Hamlett, Marx, \& Blumer, 1992). This was the only identified study, which used standardized pain and comfort scores. Overall intubation conditions were better in the remifentanil group as compared with the morphine group. One limitation was that the anesthesiologist who performed the intubation, though blinded, self-rated the complexity of the intubation. In addition, the intubation conditions (poor, good, and excellent) were subjective measurements. No excellent conditions were seen in the morphine group. This was most likely due to the time of dosing, less than 3 minutes prior to intubation, and using a drug with a peak effect of greater than 15 minutes. More than one attempt at intubation was required only in the morphine group. There were no statistically significant differences in pain or comfort scores. There were also no differences in any hemodynamic variables.

VanLooy, Schumacher, and Bhatt-Mehta (2008) developed a continuous quality improvement practice initiative to evaluate the efficacy and safety of a standardized, stepwise escalation of sedation and analgesia for nonemergent intubations in the NICU. A progressive algorithm provided initial sedation (midazolam) and analgesic ( $2 \mathrm{mcg} / \mathrm{kg} / \mathrm{dose}$ of fentanyl) with an option for a short-acting paralytic (rocuronium) when necessary. No data was collected prior to this change in practice. Heart rate, oxygen saturation, respiratory rate, mean arterial blood pressure, and pain scores were recorded at the bedside prior to, during, and for 2 hours after the intubation procedure. Physiologic data during the laryngoscopy and until the time of taping were obtained from the bedside cardiorespiratory monitor. The number of attempts made, time to successful intubation, and medications used were documented. Ninety infants were included in the change initiative. Data was available for a total of 66 intubation attempts. Nearly 80 percent of the infants required 3 attempts or fewer for successful intubation. The median number of intubation attempts was two, with the mean time for successful intubation about 7 minutes. 
Heart rate decreased and oxygen saturation increased significantly from baseline to completion of the procedure. Mean arterial blood pressure showed no significant difference. No patient received rocuronium. The investigators concluded that this systematic approach to premedication for intubation successfully prevented acute physiologic changes. There were three infants who had chest wall rigidity associated with the Fentanyl dosing. This has been reported and is generally associated with rapid administration.

In a double blind, randomized controlled trial, Choong et al. (2010) evaluated the efficacy and safety of remifentanil as a premedication for 30 hemodynamically stable term and preterm infants undergoing elective endotracheal intubation. Infants in the intervention group received $3 \mathrm{mcg} / \mathrm{kg}$ remifentanil and a normal saline placebo. Those in the control group received $2 \mathrm{mcg} / \mathrm{kg}$ fentanyl and $2 \mathrm{mcg} / \mathrm{kg}$ succinylcholine. Both groups also received $20 \mathrm{mcg} / \mathrm{kg}$ of atropine as part of the premedication regimen. The primary outcome was time to successful intubation as measured by total time in seconds from the first insertion of the laryngoscope blade in the mouth until final confirmation of endotracheal tube placement by clinical examination. Secondary outcomes were oxygen saturations, heart rate, and blood pressure changes during the procedure; time to return of spontaneous respirations; adverse events; and intubation conditions. There were no significant differences between groups in time to successful intubation, number of attempts, or degree of heart rate and saturation changes. Four infants in the remifentanil group received additional succinylcholine for intubation; one for chest wall rigidity and desaturation, another for persistent spontaneous respirations despite premedication, and two for repeated intubation attempts. Return of spontaneous respirations was longer in infants who received succinylcholine. Two infants in the remifentanil group experienced chest wall rigidity requiring 
pharmacologic intervention. Intubation conditions were not statistically significant between the 2 groups.

Most recently, Venkatesth and colleagues (2010) prospectively evaluated adverse events in 93 elective intubations in 3 NICUs in the United Kingdom over a 3 month time frame. Their premedication protocol included dosing with morphine, suxamethonium, and atropine. Data collection included: time to successful intubation, number of attempts, experience of person performing the intubation, and physiologic variables including heart rate, blood pressure, and oxygen saturations. As they used a combination of drugs, the median time to prepare the medications was 16 minutes. This might be acceptable for elective intubation, but the time delay would not be acceptable for semi-urgent intubations. Most first attempts at intubation were performed by inexperienced residents. Fifty seven percent were successful on the first attempt; $63 \%$ by the second attempt. The mean time to successful intubation was 5 minutes. Desaturations below $85 \%$ and bradycardia occurred in at least $25 \%$ of the intubations. This study highlights the high incidence of side effects of the intubation procedure even with premedication. More research needs to be done with combination of drugs and the experience of the person performing the intubation.

\section{Synthesis}

In the 8 years since Shah and Ohlsson's systematic review on the effectiveness of premedication for endotracheal intubation, little progress has been made. Limited studies with small sample size are available to evaluate efficacy and safety of different drugs used for premedication. Though the studies and drug regimens are all different, the evidence is homogenous in that awake intubation is associated with adverse physiologic changes and the need for more frequent attempts and longer time to successful intubation. Current evidence 
suggests that for routine semi-urgent intubation of neonates, the use of a premedication is a more effective technique with less potentially harmful physiological fluctuation than traditional awake intubation.

\section{Current Recommendations}

Pre-emptive treatment prior to endotracheal intubation will help ameliorate the short-term physiologic responses of the infant (American Academy of Pediatrics \& Canadian Paediatric Society, 2006; Anand, 2007; Barrington, Finer, \& Etches, 1998; Duncan, Zurick, \& Wolf, 2001). Carbajal, Eble, and Anand (2007) note that awake intubation continues to be a common practice, despite evidence that neonatal intubation is painful and stressful. They suggest that tracheal intubation without the use of sedation or analgesia should only be performed for emergent intubations in the delivery room. Current recommendations from the American Academy of Pediatrics and the Canadian Pediatric Society (2006) include the administration of an opioid as a preferable choice for premedication prior to intubation because of the rapid onset of action. The Section of Anesthesiology and Pain Medicine and the Committee on the Fetus and Newborn (2007) within the American Academy of Pediatrics began working together to create a statement on premedication for non-emergency intubation in the neonate. The AAP's recent clinical report (Kumar, Denson, Mancuso, Committee on Fetus and Newborn \& Section on Anesthesiology and Pain Medicine, 2010) advises that except for emergent intubations during resuscitation, premedication should be used for all endotracheal intubations in newborns. In addition, they recommend that these infants should receive at minimum either an analgesic or an anesthetic dose of a hypnotic. Vagolytics and rapid onset, short-acting muscle relaxants should be considered. Because of the variability in dosing and the existing data to this point, patients in the NICU at West Virginia University Children's Hospital were not routinely receiving 
premedication prior to the intubation procedure. The purpose of this evaluation of the protocol was to determine if an educational program with NICU providers would change their understanding of neonatal pain and if the protocol change to premedicate would decrease the time to successful intubation, decrease the number of intubation attempts, and decrease the incidence of adverse physiologic events.

\section{Congruence with Organizational Strategic Plan}

The implementation of this project met the mission, values, and goals of West Virginia University Hospitals (WVUH), West Virginia University Children's Hospital (WVUCH), and the West Virginia University Health Science Center (WVUHSC). All of the named organizations aim to provide state-of-the-art health care to the people of West Virginia as well as to improve the health of all populations and communities (WVUHS, 2008; WVUHSC, 2008). Each entity provides for the education of health professionals in service, research, and patient care. This protocol change with standardized dosing was implemented in the NICU at WVUCH, a children's hospital within WVUH. Providers in the NICU are employees of WVUH or WVUHSC.

Pain prevention for intubation is a standard of care in all populations but neonates. Despite the accumulating evidence that neonatal procedural pain is harmful, treatment for painful procedures such as intubation is most often not provided to the neonate. The vision of this protocol change was to premedicate all neonates prior to semi-urgent intubation attempts to provide excellence in pain prevention and state-of-the-art health cares for this vulnerable population. In addition, understanding the current pain prevention research in the newborn infant will enhance the education of all caregivers in the NICU. 


\section{Project Objectives}

Based upon the NICUs protocol, the efficacy of the evaluation of the change in protocol was to document that:

1. There will be an increase in staff knowledge, beliefs, and attitudes regarding neonatal procedural pain and treatment from pre to post education.

2. $80 \%$ of neonates requiring elective or semi-urgent endotracheal intubation in the NICU will be premedicated with one $2 \mathrm{mcg} / \mathrm{kg}$ dose of fentanyl.

3. The incidence of adverse physiologic effects during endotracheal intubation (bradycardia and desaturations) will be reduced in those neonates premedicated with fentanyl.

4. The time to successful intubation will be reduced in neonates premedicated with fentanyl.

5. There will be a reduction in the number of intubation attempts per neonate. 


\section{CHAPTER III METHODOLOGY}

\section{Project Design}

Dr. Janet Graeber and the NICU multidisciplinary leadership team developed and approved the premedication for intubation protocol change on February 26, 2008. The new protocol would include one $2 \mathrm{mcg} / \mathrm{kg}$ dose of fentanyl prior to all elective or semi-urgent intubations in the NICU. The Institutional Review Board identified the project as Non-human Subjects on March 2, 2009. The first step in the evaluation process of this protocol change was then the development of written guideline for caregivers in the NICU and a data collection sheet (Intubation Data Collection Tool), which was placed in all intubation boxes in the NICU pods. Input and consensus agreement from the neonatologists and neonatal nurse practitioners were used for the development of these documents.

The written guideline included the following steps (see Appendix A for Premedication Protocol Guideline). The protocol was to be initiated when a decision was made to intubate or reintubate a neonate. At this time, the neonatal nurse practitioner (NNP) or resident ordered the $2 \mathrm{mcg} / \mathrm{kg}$ fentanyl dose as per protocol for the bedside nurse to administer. While the nurse was obtaining the fentanyl from the Pyxis, the intubation equipment and personnel were gathered. The fentanyl was to be given intravenously as soon as possible; no less than one minute prior to an intubation attempt. A member of the team present at the time would be designated as an observer, data recorder, and timekeeper. This person documented on the Intubation Data Collection Tool (see Appendix B): (a) the time at decision to intubate, (b) the time the fentanyl was given (post-initiation of the protocol), (c) the time the laryngoscope was placed in the infant's mouth, (d) the time of successful intubation, (e) the number of attempts at intubation, and (f) the experience of the person attempting and/or completing the intubation procedure. The 
total time taken to complete intubation was defined as the interval from when the laryngoscope was placed in the neonate's mouth to successful laryngeal insertion of the endotracheal tube. A continuous monitor strip was to be printed for the time frame five minutes prior to the intubation attempt to five minutes after successful intubation was completed and attached to the Intubation Data Collection Tool. As the monitor strip graphed the physiologic variables without specific numbers, 15- second intervals of heart rate and saturations for the planned time period were retrieved from the NICU central monitor within 24 hours of the intubation procedure. If the intubation was emergent, the reason was documented on the Intubation Data Collection Tool. These were labeled and placed in a box in the NNP office after each intubation.

A variety of stakeholders were identified. The multidisciplinary leadership team of the WVU Children's Hospital NICU supported and approved this change project. This team consists of six neonatologists, 9 NNPs, and the NICU nurse manager. Other stakeholders included all nursing staff in the NICU, all respiratory therapists that work in the NICU, and small groups of residents that rotate through the NICU monthly. The ultimate stakeholder was the neonate undergoing the intubation procedure

The general focus was to guide the stakeholders toward the understanding of the current science and practices in neonatal procedural pain, including intubation, as well as gain the acceptance that premedication is an ethical practice in the NICU. The direct focus was a permanent change in practice, reflecting excellence in patient-focused care. Promotion and advertisement were the main strategies used to facilitate focus for the stakeholders.

After the written protocol guideline and the documentation sheets were finalized, change champions were identified and educated. Initial promotional material was a poster (previously designed for this project) placed in the NICU lounge. Stakeholders were notified of the change 
in protocol via email and the use of bulletin boards in the NICU lounge, resident workroom, respiratory therapy lounge, and the NNP office.

Next, education and training of all stakeholders took place. Promotional materials (posters, signs) and educational materials (verbal, written, and visual) were presented to all stakeholders who attended one session during their scheduled work hours (Appendix C for complete curriculum outline). These sessions served two purposes: (a) understanding why premedication for intubation is necessary and (b) provision of skills to implement the change. Pre- and posttests were given to all stakeholders to assess basic knowledge of procedural pain and treatment; attitudes and beliefs on neonatal pain; and the effectiveness of the educational sessions (Assessment of Knowledge, Beliefs, and Attitudes about Infant Procedural Pain, see Appendix D). This tool was adapted with permission from the Pediatric Nurses' Knowledge and Attitude Survey Regarding Pain (PNKAS) (Ferrell \& McCaffery, 2008) and the Infant Pain Questionnaire (Porter et al., 1997). The NNPs and the attending neonatologists were the first groups to attend the educational sessions, as they would be the most important visual support for this project; only with their acceptance would the fentanyl routinely be given. This investigator led each session to assure consistency of information delivered. Once the education sessions were completed and the project process reevaluated, the next step was to meet with the NNP team to finalize primary tasks, roles and responsibilities. A timeline was developed and distributed to the NICU multidisciplinary leadership team.

An official start date was identified and promoted with signage in the NICU lounge and the NNP office. An announcement was sent to all stakeholders via email and postcards were placed in the mailboxes of the NICU nurses and respiratory therapists. 


\section{Instruments}

The modified PNKAS is a self-administered 20-item inventory that includes 15 true/false questions about general knowledge of neonatal pain and 5 question regarding beliefs about neonatal pain and treatment. The test takes about 15 minutes to complete. Content validity of the original test had been established by a review of a national nursing panel of pediatric nursing experts as well as review by Betty Ferrell, the author of the original tool. Test-retest reliability $(r>$.80) and internal consistency reliability (alpha $r>0.70)$ were both established for the original test. As the PNKAS was modified for this evaluation and the number of questions was significantly reduced, a Cronbach's alpha was not calculated for this adapted test.

\section{Project Timeline}

The key to successful completion of any project is planning, setting goals, and establishing a project timeline. The timeline for this project is delineated in Appendix E. It was estimated that the time from development of the written protocol, documentation tool, and education materials to completion would be approximately 7 months. Due to other time constraints, there was a time lag between the planning of this change and actual implementation. Data collection occurred over a 6 month period.

\section{Resources}

A variety of resources were used to support the evaluation of the efficacy of the change in protocol. Personnel resources were the strongest and most important advantage to the acceptance of this new intervention in the NICU. The investigator oversaw the implementation of the evaluation. A letter documenting key site support is included as Appendix F. Eight change champions were chosen from multiple levels of care givers to provide support and guidance to the stakeholders of the project at all levels 24 hours a day. The main areas of 
coverage by these support persons were the implementation of procedure and completion of the documentation forms. Effective two-way communication, the foundation of any successful project, was necessary throughout the project.

With over 130 stakeholders in this change project, a complete communication plan was necessary to support and continue buy in for this project. A variety of channels were used including email, bulletin board notices, and reminder cards in mailboxes. The project coordinator and the change champions were available on a variety of shifts on a regular basis to provide feedback opportunities, answer questions, and reinforce key messages.

\section{Financial Plan/Budget}

A simple, but detailed budget was developed for this project (see Appendix G). Project expenses were minimal. The Division of Neonatology and the School of Nursing provided nonmonetary support for copying, copy paper, and an educational poster. The only start up expenses were for additional copy paper and card stock. These were used for initial and ongoing education and communication with the multidisciplinary providers in the NICU. Organizational expenses include hospital equipment necessary to administer a dose of fentanyl. The TwinPack is a needleless device used to withdraw the fentanyl from the vial. Two syringes and an extra needle are used, as the fentanyl needs to be diluted after drawing it out of the original vial. No additional expenses were identified. Education time for all caregivers was completed during their work time as approved by the NICU nurse manager. Education for the neonatal nurse practitioners was at a regularly scheduled meeting. The resident staff were educated only when they were on the NICU rotation. No direct income was generated with the implementation of this project. There was potentially a cost savings in the number of endotracheal tubes used (\$1.74 per tube) but this was not calculated. The charge to the patient for one dose of fentanyl 
was $\$ 13.29$. In summary, though no known short-term or long-term cost benefits were associated with implementing premedication with intubation in the NICU, it was ethically a sound practice change.

\section{Planned Evaluation}

Two evaluation focal areas were identified: programmatic focus and effectiveness of the program in reducing adverse physiologic effects of intubation, time to successful intubation, and number of intubation attempts.

Programmatic evaluation was maintained throughout the entire evaluation project. To assess the effectiveness of the caregiver education programs as well as staff beliefs and attitudes regarding procedural pain in the neonate, a pretest and posttest was given to all stakeholders before and after the education sessions. A paired t-test and ANOVA were used to evaluate possible differences in knowledge, beliefs, and attitudes within and between groups.

A weekly review of the number of non-delivery room intubations performed in the NICU was obtained from the NICU electronic medical record system, Site of Care. Comparison of this total with the number of Intubation Data Collection Tools completed assessed whether documentation forms were being completed on every intubation procedure. In addition, the percentage of neonates being premedicated prior to intubation was calculated. Assessment of full completion of the Intubation Data Collection Tools was assessed weekly. Obstacles to premedication could be ascertained from this information.

The evaluation plan for determining the primary effectiveness of premedication with fentanyl prior to intubation was to compare the incidence of primary adverse physiologic effects (bradycardia and desaturations) pre and post-implementation of the change in protocol. In addition, differences in the time to successful intubation were calculated from documented 
intubation procedure times, and the numbers of attempts per neonate during intubation were tallied. T-tests and ANOVA were used to compare time to successful intubation, actual number of intubation attempts, and changes in heart rate and oxygen saturations before, during, and after the intubation procedure. Demographic data were collected. Independent t-tests were used to compare demographic data such as: gestational age, age at intubation, weight at intubation, and indication for intubation. 


\section{CHAPTER IV RESULTS}

\section{Assessment of Knowledge, Beliefs, and Attitudes of NICU Caregivers}

\section{Demographics}

A total of $53(48 \%)$ of all involved staff completed both the pre-and post-PNKAS. The sample was predominantly female (92\%) staff nurses $(66 \%)$. Over half of these staff nurses $(68 \%)$ were baccalaureate-prepared. Other roles included neonatal nurse practitioners (8), respiratory therapists (5), neonatal fellows (2), and attending physicians (3). The mean age was 36.74 years with a range from 20 to 59 years. The mean number of years of experience was 12.68 years, though the range was $0-36$ years.

\section{Results of Pre- and Post test}

Scores were expressed as percentage of correct responses. Differences in scores between and among groups were determined either by paired sample t-test or analysis of variance (ANOVA). The mean corrected score for the posttest 13.81 (92\%) was significantly higher than the pretest $(81 \%)(\mathrm{p}<.003)$. These results revealed a high level of knowledge across role, experience, and educational level in this NICU. There were no differences in the mean increase in score within groups but a significant difference was seen between groups $(p<.019)$ and between educational levels $(\mathrm{p}<.001)$. Master's degree NNPs scored significantly higher than respiratory therapists $(\mathrm{p}<.019)$. NNPs and MDs scored higher than ADN-prepared nurses $(\mathrm{p}<.009 ; \mathrm{p}<.029)$. There was no significant difference among the scores of the BSN-prepared nurses, NNPs, and physicians.

Participants were asked to rate the intensity of the infant's pain during a variety of procedures in comparison with adults. All but 2 people completing the survey (96\%) believed 
that infants felt as much pain as adults, if not more. The remaining 2 people believed that an infant felt less pain than adults during chest tube placement and circumcision. There was lack of knowledge about current provision of analgesics during circumcision in this NICU. Ten percent of nurses incorrectly believed that no analgesics or penile blocks were provided for circumcision, when in actuality premedication is provided $100 \%$ of the time in the NICU.

\section{Premedication for Intubation}

\section{Infant Enrollment and Demographics}

Between December 1, 2009 and May 31, 2010, there were a total of 107 non-delivery room intubations documented in the electronic medical record in the NICU at WVUCH. Data collection tools were completed for 89 infants ( $83 \%$ of total intubations). The NICU staff decided if the intubation was emergent, semi-urgent, or elective. Five infants were excluded for a variety of reasons. One infant was on a fentanyl drip at the time of intubation and one received fentanyl after multiple attempts at intubation. One infant had a significant adverse affect to the fentanyl. Immediately following dosing, the infant became bradycardic with saturations below $60 \%$. Chest wall rigidity was documented as the cause of the event, but when the physiologic data was reviewed, the infant's heart rate and saturations immediately improved when the infant was intubated. The nurse reported that she gave the dose by rapid bolus instead of slow infusion over 1 minute as should be standard procedure causing significant respiratory depression. This infant was excluded from any future premedication with fentanyl for intubation. Data was unable to be retrieved from the central monitor on 2 infants. Only 6 intubations were actually missed. Three of these missed intubations occurred during the premedication phase of the evaluation so $92 \%$ of eligible infants received fentanyl prior to intubation. Thus, 78 infants undergoing elective or semi-urgent intubation were evaluated during the program change. Fifty 
of the infants were assessed undergoing awake intubation; 38 infants were given fentanyl prior to the intubation procedure. After review of physiologic data on all infants, 23 were not included in analysis because of physiologic instability in the 5 minutes prior to intubation. This was defined by the investigator as severe hypoxemia with saturations $<80 \%$ and/or heart rate less than 80 beats per minute for more than 3 minutes prior to the procedure. These were emergent intubations. The remaining 55 infants were included in analysis of the protocol change project. Twenty four infants received fentanyl prior to intubation; 31 received no medication. There were no significant differences in baseline demographics between groups, which are summarized in Table 1.

Table 1 Infant Demographic Characteristics

\begin{tabular}{|c|c|c|c|c|c|}
\hline & Drug & $\mathrm{n}$ & $\mathrm{M}$ & SD & $\mathrm{p}$ \\
\hline GA weeks & $\begin{array}{l}\text { no } \\
\text { yes }\end{array}$ & $\begin{array}{l}31 \\
24\end{array}$ & $\begin{array}{l}27.94 \\
28.21\end{array}$ & $\begin{array}{l}5.215 \\
5.283\end{array}$ & ns \\
\hline Days & $\begin{array}{l}\text { n o } \\
\text { yes }\end{array}$ & $\begin{array}{l}31 \\
24\end{array}$ & $\begin{array}{l}35.26 \\
32.08\end{array}$ & $\begin{array}{l}39.816 \\
36.643\end{array}$ & ns \\
\hline Birth wt grams & $\begin{array}{c}\text { no } \\
\text { yes }\end{array}$ & $\begin{array}{l}31 \\
24\end{array}$ & $\begin{array}{l}1162.84 \\
1248.49\end{array}$ & $\begin{array}{l}1026.024 \\
993.157\end{array}$ & ns \\
\hline Intubation wt grams & $\begin{array}{l}\text { no } \\
\text { yes }\end{array}$ & $\begin{array}{l}31 \\
24\end{array}$ & $\begin{array}{l}1536.87 \\
1681.92\end{array}$ & $\begin{array}{l}893.347 \\
893.261\end{array}$ & ns \\
\hline
\end{tabular}

In this Level 3 NICU, 91\% of all infants either had respiratory distress syndrome (RDS) or bronchopulmonary dysplasia. The remaining $9 \%$ had a variety of non-RDS disease processes such as meconium aspiration syndrome. 


\section{Results for Intubations Relative to Time, Attempts, and Adverse Effects}

The most common reason for intubation during this time period was deterioration in respiratory status (53\%) followed by "accidental” extubation (27\%) and elective endotracheal tube placement (20\%). Neonatal nurse practitioners intubated $51 \%$ of infants while residents and transport nurses intubated the remaining infants, with a fairly equal distribution between those infants who received premedication and those who were not premedicated. Fifty five percent of intubations were successful on the first attempt; $20 \%$ on the second attempt. There were no significant differences among the role of the person who performed the intubation, the disease process, and whether the infant did or did not receive premedication.

There were no significant differences in time to intubation between those infants who received fentanyl and those who did not receive fentanyl (246 vs. 204 seconds; $\mathrm{p}<.547)$. There was a significant difference in time to intubation when comparing the NNPs with all other providers regardless of premedication as summarized in Table 2 and Table 3. For the purpose of this analysis, NNP status was dichotomized into NNP or other. This statistical difference was also seen when comparing this same parameter in all 78 infants prior to imposing the exclusion criteria (301 seconds vs. 104 seconds; $\mathrm{p}<.000)$. 
Table 2 Time to Intubation Role vs. Premedication

\begin{tabular}{|ll|l|l|l|l|}
\hline NNP indicator & Drug & $\mathrm{n}$ & Mean & SD & $\mathrm{p}$ \\
\hline NonNNP & no & 17 & 333.53 & 298.992 & $\mathrm{~ns}$ \\
& yes & 10 & 312.00 & 348.195 & \\
\hline NNP & no & 14 & 141.43 & 150.543 & $\mathrm{~ns}$ \\
& yes & 14 & 127.50 & 128.598 & \\
& & & & & \\
\hline
\end{tabular}

Table 3 Time to Intubation NNP vs. NonNNP

\begin{tabular}{|l|l|l|l|l|}
\hline & $\mathrm{n}$ & Mean & SD & $\mathrm{p}$ \\
\hline NonNNP & 27 & 325.56 & 311.597 & $\mathrm{P}<.007$ \\
& 28 & 134.46 & 137.567 & \\
\hline
\end{tabular}

There was no significant difference in mean number of intubation attempts between the infants who received fentanyl and those who were not premedicated (1.83 vs. $2.26 ; \mathrm{p}<.290$ ). The neonatal nur se practitioners required fewer m ean attempts for s uccessful intubation than ot her providers as seen in Table 4. Longevity in this NNP c ohort ranged from 1 year to 30 years. There was no difference in successful intubation by NNP experience level.

Table 4 Number of Intubation Attempts NNP vs. NonNNP

\begin{tabular}{|l|l|l|l|l|}
\hline & $\mathrm{n}$ & Mean & SD & $\mathrm{p}$ \\
\hline NonNNP & 27 & 2.56 & 1.649 & $\mathrm{P}<.013$ \\
NNP & 28 & 1.61 & 1.100 & \\
\hline
\end{tabular}


There were also no significant differences in heart rate and oxygen saturation changes between the group of infants who received fentanyl and those not receiving fentanyl as well as between provider groups. Average saturations during the measured time frame were similar between the premedicated $(85 \%)$ and nonmedicated infants $(90 \%)(p<.276)$ as well as between the NNP group and all other groups $(90 \%$ vs. $85 \%, \mathrm{p}<.198)$. Mean minimum saturations in all groups ranged from 70-77\%. Mean minimum heart rates in all groups ranged from 121-130 beats per minute. Many infants had a brief desaturation and heart rate drop within the first 2 minutes after the endotracheal tube was placed. This may have been due to a vagal response or deep placement of the endotracheal tube prior to securing with tape. Though the infants experienced short episodes of desaturations, they experienced no prolonged episodes of hypoxia or hyperoxia which can be detrimental to maturing body systems. 


\section{CHAPTER V SUMMARY, DISCUSSION, IMPLICATIONS}

\section{Congruence with Theoretical Model}

The Theory of Symptom Management (TSM) was used as a guide for the development of an educational program for NICU providers about neonatal pain and an evaluation of a protocol change to premedicate prior to intubation in the NICU. One concept of this model is that symptoms do not have to be experienced by an individual, only be at risk of developing the symptom. Management strategies are then developed to prevent the symptoms or negative outcomes through the intervention. It is well known that intubation is a painful procedure in older children and adults. As neonates have similar adverse affects during the intubation procedure, it is presumed that they experience pain as well. The development of the standard protocol to provide fentanyl prior to intubation targeted a group of neonates at risk of experiencing pain to decrease the adverse physiologic effects associated with the procedure. This in turn would decrease the time to successful intubation and the number of attempts In the NICU environment, it is the nurse who interprets the need for pain medication, based on physiologic and behavioral cues of the infant. The volume of caregivers in the NICU limits intimate knowledge of an individual infant's specific cues and thus may decrease needed treatment of pain in this population. Again, a standardized treatment protocol assures preemptive treatment of potential pain.

The education program for all caregivers provided information on the historical perspectives in recognition and treatment of neonatal procedural pain, as well as current recommendations for pharmacologic treatment prior to intubation. A standardized program of education minimizes misconceptions of procedural pain, inaccurate assessments, and missed opportunities for treatment. 
In summary, TSM provided a framework for evaluating symptoms in the nonverbal population of the NICU, preventing known adverse effects of the intubation procedure by proactively giving fentanyl prior to the procedure.

\section{Discussion}

The purpose of this project was to evaluate a protocol change to determine if an educational program with NICU caregivers would change their understanding of neonatal pain. The second purpose was to determine if the protocol change to premedicate prior to intubation would decrease the time to successful intubation, decrease the number of intubation attempts, and decrease the incidence of adverse physiologic events.

\section{Assessment of Knowledge, Beliefs, and Attitudes of NICU Caregivers}

Knowledge and attitudes about neonatal pain were examined in multiple different caregivers in the NICU. This was not compared to their actual practice of pain assessment or their practice of providing pain treatment interventions.

McCaffery chose a score of $>80 \%$ as passing and nurse educators often consider $85 \%$ acceptable for competency testing (Ferrell \& McCaffery, 2008). She felt that if nurses scored less than that, the ability to care for patients in pain was significantly compromised. In general, the test results of NICU caregivers revealed a high level of knowledge across role, experience, and educational level in this NICU. No generalized knowledge deficiencies were identified. This may have occurred because general pain management and treatment are a priority of this NICU and are discussed on daily rounds for individual patients. In addition, all nursing staff received education on pain management during their orientation. As noted, respiratory therapists scored significantly lower than Master's degree NNPs and MDs. These therapists do not receive any education about pain management and treatment. They should be included in future 
inservices and educational updates. The BSN-prepared nurses scored as high as the NNPs and physicians. This may indicate adequacy of pain education in basic baccalaureate-preparation.

The scores on this test may have been even higher, as the majority of those taking the test answered one question incorrectly. This question asked whether infants can be distracted from severe pain. The question may have been confusing to those who practice in the NICU where critically ill infants are not easily distracted and sleep for long periods in the day.

One significant limitation was that only $48 \%$ of caregivers completed both pre-and posttest. As staff attended the educational session during their shift, many had to return to patient care prior to completing the posttest. This response rate may bias the findings. After the results of this change program are communicated to the NICU, it would be helpful to resurvey all staff to assess knowledge and beliefs about neonatal procedural pain to assist in the development of future educational programming.

It was concerning that there was inconsistent knowledge about current provision of analgesics for painful procedures in the NICU. Clearly, more education across caregivers is needed about premedication for other procedures.

\section{Infant Enrollment and Premedication}

Only 6 of the 107 non-delivery room intubations were missed for evaluation. Three of these missed intubations occurred during the premedication phase of the evaluation so $92 \%$ of eligible infants received fentanyl prior to intubation. The reason noted for all missed intubations was that the caregivers did not remember until after the intubation was successful. The success for patient inclusion was two-fold. The data collection tools placed in all intubation boxes were brightly colored half sheets of paper that were immediately seen when these boxes were opened. It was a visible prompt when staff went to obtain the laryngoscope and the endotracheal tube. In 
addition, the data collection tool required very little documentation time by the NICU caregivers. All demographic and physiologic data were retrieved at a later time by the investigator. Though most staff nurses and respiratory therapists were in agreement with this practice change, it was the support of the NNPs that prompted its success. They were diligent in remembering and directing the nursing staff to fill out the data collections tool and obtain the fentanyl after the change was initiated. Retrospectively, when reviewing the number of infants identified but not included in the analysis because of physiologic instability prior to intubation, the NNPs may have been overzealous in determining what constituted an elective or semi-urgent vs. urgent intubation.

\section{Results for Intubations Relative to Time, Attempts, and Adverse Effects}

There were no significant differences in time to intubation, number of attempts, or incidence of adverse physiologic effects between those infants who received fentanyl and those who did not receive fentanyl. These findings are inconsistent with most similar studies evaluating the use of premedication for intubation (Dempsey, Al Hazzani, Faucher, \& Barrington, 2006: Ghanta et al., 2006; Hassad et al., 2007; Naulaers, Deloof, Vanhole, Kola, \& Devlieger, 1997; Oei, Butha, \& Lui, 2002).

This protocol change compared one drug, fentanyl, to awake intubation. Lemyre and colleagues (2004) were the only other investigators to compare one analgesic, morphine, to awake intubation. Their study failed to demonstrate the effectiveness of morphine in reducing physiologic instability or time to performing elective intubations. The authors concluded that the peak action of morphine (15-30 minutes) was perhaps too long to use for a procedure such as intubation, as it would not provide sufficient relaxation. They recommended a more rapid onset 
analgesic such as remifentanil. It may be that use of just a narcotic will not alleviate the adverse effects of this procedure.

One significant difference between this evaluation and all other studies is that those studies evaluated intubations performed under controlled conditions e.g. elective or semi-elective intubations. Conditions for intubations in this evaluation were not controlled. The decision to premedicate was at the discretion of the person performing the intubation. Therefore, some of the intubations may have been misclassified as semi-urgent. Deterioration in respiratory status and accidental extubations accounted for $80 \%$ of all intubations in this evaluation and 23 intubations were not included in analysis because they were emergent, with significant physiologic instability in the 5 minutes prior to intubation.

Only one prior study controlled for the role of the person performing the intubation (Bhutada et al., 2000). Two experienced fellows performed all intubations. In this study, in contrast to Bhutada, there was substantial variability in the level of experience between individuals performing the intubations. One significant finding was that time to successful intubation and numbers of attempts required were significantly less in the NNP group independent of whether premedication was used. Though experience of the person performing the intubation has been highlighted in other studies, to date, no studies have compared the experience of the neonatal nurse practitioner to other roles in the NICU, particularly resident physicians. It may be argued that restricted intensive care experience in pediatric residencies has impacted procedural competence but this has not been studied. In a community hospital, a general pediatrician is responsible for managing resuscitation in the delivery room and stabilizing an ill infant. It is then important that the pediatrician be proficient in intubation and that this competency-based learning be emphasized in pediatric residency training. Though the 
NNP supervises almost all intubations in this NICU, a consistency in directed support at the bedside when the resident is attempting the intubation should be examined further.

\section{Summary of Protocol Change}

In summary, all of the objectives of this protocol change were not achieved. Though more than $80 \%$ of infants requiring intubation in the NICU were premedicated with fentanyl after the protocol change, there was no difference in time to successful intubation, number of intubation attempts, or variation in physiologic variables. Fentanyl is a narcotic analgesic that would not alleviate or attenuate vagal adverse effects. In addition, the discomfort of the positioning and pressure of the laryngoscope in the pharynx may also precipitate movement during the procedure, limiting an adequate view of the epiglottis, particularly in an inexperienced intubator. Any of these adverse effects as well as the urgency of many of the intubations may affect time to successful intubation. There were no significant adverse effects during the fentanyl dosing except for the one infant who experienced respiratory depression following rapid infusion of the medication.

Nursing staff and respiratory therapists actively supported this protocol change. They were instrumental in the timely acquisition of the fentanyl dose and documentation of data. Any further programmatic change would require their active support.

Premedication for endotracheal intubation is evolving as a standard of care for infants in the NICU. Providing excellence in pain prevention and state-of-the-art health cares for this vulnerable population continues to be congruent with the mission of WVUCH and the NICU. The most appropriate selection of a drug and safety of medication use are still unclear. This evaluation of the protocol change adds to the growing body of evidence on the use of premedication for intubation in the neonatal population. 


\section{Recommendations}

As a result of the protocol change program, several recommendations can be made for further intervention to promote premedication for procedural pain such as intubation in the NICU. A lower dose fentanyl was chosen as the sole drug for premedication as the staff in the NICU was familiar and comfortable with dosing. In addition, there is a known increased incidence of respiratory depression and chest wall rigidity with higher doses and rapid infusion. After presenting this information to the NICU leadership team, a recommendation for dosing would include the same dose of fentanyl in conjunction with a short-acting paralytic or sedative. Fentany remains the opiate of choice because of its fast onset of action and relative cardiac stability. The current dose is one recommended by the American Academy of Pediatrics (Kumar, Denson, Mancuso, Committee on Fetus and Newborn \& Section on Anesthesiology and Pain Medicine, 2010). Using fast acting drugs in adequate doses may create a deep hypnotic state that would facilitate faster, pain free intubations. The potential for adverse side effects can be reduced by slow administration over at least one minute.

A specific practice guideline with regard to procedural premedication should be developed. After this guideline is developed and education sessions held for all caregivers in the NICU, ongoing monitoring of procedural premedication should be conducted quarterly. It is recommended that a multidisciplinary team monitor these outcomes.

Although there is a need to provide sufficient procedural skills to pediatric residents, a structured oversight during the procedure may facilitate a more successful attempt in less time for this group. This would need to be actively supported by the NNP team. 


\section{Additional Data}

Though the leadership team in the NICU has had concerns about the frequency of "accidental" or unplanned extubations in this NICU, there was no prior data to support this premise. Of the initial 78 intubations analyzed in this sample, $40 \%$ were caused by unexpected or accidental extubations. This is most often a preventable cause, such as poor securement to the face or the nurse's inattentiveness to tube placement and head position.

Accidental extubations often lead to emergent, less-controlled endotracheal reintubations. Repeated intubations, especially those performed emergently, increase the risk of laryngeal or tracheal injury and scarring, pulmonary injury from excessive ventilation, ventilator-associated pneumonia, and nonpulmonary complications such as intraventricular hemorrhage (McMillian et al., 1989). The results of this study have been given to the neonatal nurse clinician in the NICU. The procedure for taping and securing the tube has been changed and a quality improvement program is being developed. 


\section{Appendix A}

\section{Premedication Protocol Guideline}

- The guideline will be initiated when the decision is made to intubate or reintubate any neonate

- Gather equipment and personnel

- $\quad \mathrm{NNP}$ order $2 \mathrm{mcg} / \mathrm{kg}$ fentanyl

- Bedside nurse will obtain fentanyl from Pyxis

- Fentanyl given IV as soon as possible; no less than one minute prior to an intubation attempt

- Clinically uninvolved member staff present will be designated as an observer, recorder, and, timekeeper

o Time at decision to intubate

o Time laryngoscope placed in neonate's mouth

o Time at successful intubation

o Number of attempts

o Experience of person attempting and/or completing procedure

- If the neonate is felt to be too unstable to wait the extra time to obtain the fentanyl, the reason will be documented on the data collection tool.

- When completed, the data collection tool will be placed in a designated box in the NNPs office. 


\section{Appendix B}

Intubation Data Collection Tool

Name:

Reason for intubation:

Premedication: Yes No

Person intubating:

Time Fentanyl given:

Time of successful intubation:
Date:

Time:

If no, document why not:
Time laryngoscope placed in mouth:

Number of attempts: 


\section{Appendix C}

Curriculum Outline

Premedication for Semi-urgent Endotracheal Intubation

1. Pretest: Knowledge, Attitudes, and Beliefs about Neonatal Pain

2. Historical perspective on treatment of neonatal pain

3. Neonatal general procedural pain

a. Signs and symptoms

b. Assessment

c. Current standards for treatment

4. Neonatal intubation
a. Adverse physiologic effects
b. Current literature
c. Current and future AAP recommendations

5. Implementation of change in the protocol
a. Timeline
b. Protocol
c. Individual responsibilities
d. Intubation Data Collection Tool
e. Plans for follow-up after completion of change project 


\section{Appendix D}

Assessment of Knowledge, Beliefs, and Attitudes about Infant Procedural Pain

Unit/Position:

Age:

Sex: Male Female

Years of Experience:

Educational Preparation:

1) Because of an underdeveloped neurologic system, infants less than 1 year of age have decreased pain sensitivity and limited memory of painful experiences.
a) True
b) False

2) If the infants can be distracted from the pain, this usually means that he/she is not experiencing high levels of pain.
a) True
b) False

3) Infants may sleep in spite of severe pain.
a) True
b) False

4) Non-drug interventions (swaddling, music, sugar nipples) are very effective for mildmoderate pain control but are rarely helpful for more severe pain.
a) True
b) False

5) Parents should not be present during painful procedures.
a) True
b) False

6) Infants cannot tolerate opioids for pain relief.
a) True
b) False

7) Anxiolytics and sedatives are appropriate medications for the relief of pain during painful procedures.
a) True
b) False

8) After the initial recommended dose of opioid analgesic, subsequent doses should be adjusted according to individual patient's response.
a) True
b) False 
9) Fentanyl, a synthetic narcotic has an almost immediate peak effect, though the duration can vary from 1-15 hours.
a) True
b) False

10) Chest rigidity, a side effect of Fentanyl, can be reversed with a dose of Narcan.
a) True
b) False

11) Vital signs are always reliable indicators of the intensity of an infant's pain.
a) True
b) False

12) Though many NICUs in Europe, Australia, and Canada provide pain medication prior to intubation, less than $1 \%$ of NICUs in the United States premedication prior to intubation.
a) True
b) False

13) Morphine is the drug of choice for pain during emergent procedures.
a) True
b) False

14) Intubation is painful and stressful.
a) True
b) False

15) It is ethically sound practice to provide pain medication prior to all urgent, semi-urgent, or elective painful procedures to all infants.
a) True
b) False

16) Think about the intensity of pain that infants experience and for the same painful stimulus, rate the pain the infant feels as compared with the pain an adult feels where:

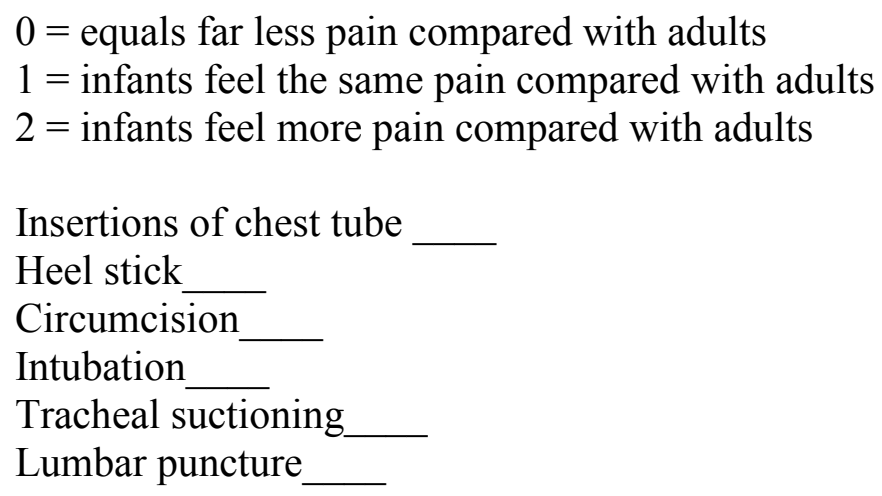


17) Rate how painful you believe each procedure is where:

$$
\begin{aligned}
& 0=\text { not painful } \\
& 1=\text { somewhat painful } \\
& 2=\text { moderately painful } \\
& 3=\text { quite painful } \\
& 4=\text { very painful }
\end{aligned}
$$

Insertions of chest tube

Heel stick

Circumcision

Intubation

Tracheal suctioning

Lumbar puncture

18) Rate how often you believe each procedure is performed with pharmacologic agents (analgesia, anesthesia) and with comfort measures (pacifier, swaddling, positioning) where:

$$
\begin{aligned}
& 0=\text { never } \\
& 1=\text { rarely } \\
& 2=\text { often } \\
& 3=\text { usually } \\
& 4=\text { always }
\end{aligned}
$$

Insertions of chest tube

Heel stick

Circumcision

Intubation

Tracheal suctioning

Lumbar puncture

19) Rate how often you believe each procedure should be performed with pharmacologic and comfort measures where:

$$
\begin{aligned}
& 0=\text { never } \\
& 1=\text { rarely } \\
& 2=\text { often } \\
& 3=\text { usually } \\
& 4=\text { always }
\end{aligned}
$$

Insertions of chest tube

Heel stick

Circumcision

Intubation

Tracheal suctioning

Lumbar puncture 
20) What information do you need about procedural pain and treatment in the NICU? 


\section{Appendix E}

Timeline

\begin{tabular}{|l|l|}
\hline February 2008 & Dr. Janet Graeber and leadership team developed change in protocol \\
& Presented proposal to evaluate protocol change to interdisciplinary \\
\hline July 2008 & Developed written protocol guideline and data collection sheet \\
& Developed written and visual education materials \\
\hline September 2008 & Capstone proposal approved \\
\hline March 2009 & Summary of program change to IRB - approved \\
\hline October 2009 & Education of all stakeholders \\
\hline December 2009 & Initiation of data collection \\
\hline May 2010 & Completion of data collection \\
\hline December 2010 & Evaluation and presentation of findings \\
\hline
\end{tabular}




\section{Appendix F}

\section{Key Site Support Letter}

April 18, 2008

To Whom It May Concern:

As the neonatal project mentor for Judi Polak's capstone project, "Premedication for Semiurgent Intubation in the NICU," I am happy to provide key site support. Ms. Polak will be conducting her work in the Neonatal Intensive Care Unit at Ruby Memorial Hospital. This project should be an important addition to the area of pain and comfort control in the neonatal population.

Sincerely,

Janet Graeber MD

Chief, Section of Neonatology

Medical Director, NICU 


\section{Appendix G}

Financial Plan/Budget

\begin{tabular}{|c|c|c|}
\hline INCOME & INCOME SOURCE & AMOUNT \\
\hline & Non-Monetary Support & $\$ c$ \\
\hline & $\begin{array}{l}\text { Department of Pediatrics: } \\
\text { Copying } \\
\text { WVU SON: Poster }\end{array}$ & \\
\hline PROJECT EXPENSE & TYPE OF EXPENSE & COST \\
\hline & Card Stock 250 sheets & $\$ 11.4 \mathrm{C}$ \\
\hline & Paper (500 sheets) & $\$ 6.79$ \\
\hline ORGANIZATION EXPENSE & $\begin{array}{l}1 \text { vial of Fentanyl } \\
(100 \mathrm{mcg} / \mathrm{ml})\end{array}$ & $\$ 0.81 \times 50$ doses $=\$ 40.50$ \\
\hline & 1 B-D TwinPack & $\$ 0.28 \times 50=\$ 14.00$ \\
\hline & $1(1 \mathrm{ml})$ syringe & $\$ 0.49 \times 50=\$ 24.50$ \\
\hline & $1(10 \mathrm{ml})$ syringe & $\$ 0.71 \times 50=\$ 35.50$ \\
\hline & Kendall 18 gauge needle & $\$ 0.26 \times 50=\$ 13.00$ \\
\hline & Alcohol wipes & $\$ 0.02 \times 50=\$ 1.00$ \\
\hline TOTAL PROJECT EXPENSE & & $\$ 146.78$ \\
\hline
\end{tabular}




\section{References}

Als, H., Lawhon, G., Duffy, F. H., McAnulty, G. B., Gibes-Grossman, R. \& Blickman, J. (1994). Individualized developmental care for the very low birth weight preterm infant: Medical and neuro-functional effects. Journal of the American Medical Association, $272,853-858$.

Ambuel, B., Hamlett, K. W., Marx, C. M., \& Blumer, J. L. (1992). Assessing distress in pediatric intensive care environments: the COMFORT score. Journal of Pediatric Psychology, 17, 95-109.

American Academy of Pediatrics (2007). Section on anesthesiology and pain medicine newsletter. Retrieved July 11, 2008 from http://www.aap.org/section/anes/AnNewsfall07

American Academy of Pediatrics \& Canadian Paediatric Society. (2006). Prevention and management of pain in the neonate: an update. Pediatrics, 118, 2231-2241.

Anand, K. J. (2007). Pharmacologic approaches to the management of pain in the neonatal intensive care unit. Journal of Perinatology, 27, S4-S11.

Anand, K. J., Phil, D., \& Hickey, P. R. (1987). Pain and its effect in the human neonate and fetus. New England Journal of Medicine, 317, 1321-1329.

Barrington, K. J., Finer, N. N., \& Etches, P. C. (1998). Succinylcholine and atropine for premedication of the newborn infant before nasotracheal intubation: A randomized controlled trial. Critical Care Medicine, 17, 1293-1296.

Bhutada, A., Sahni, R., Rastogi, S., \& Wung, J. (2000). Randomised controlled trial of thiopental for intubation in neonates. Archives of Diseases in Childhood Fetal and Neonatal Edition, 82, 34-37 
Blackburn, S. (1998). Environmental impact of the NICU on developmental outcomes. Journal of Pediatric Nursing, 13, 279-289.

Bonow, F. P., Piva, J. P., Garcia, P. C., \& Eckert, G. U. (2004). Assessment of intubation procedures at reference pediatric and neonatal intensive care units. Journal of Pediatrics, $80,355-362$.

Buskila, D., Neumann, L., Zmora, E., Feldman, M., Bolotin, A., \& Press, J. (2003). Pain sensitivity in prematurely born adolescents. Archives of Pediatrics and Adolescent Medicine, 157, 1079-1082.

Buttner, W. \& Finke, W. (2000). Analysis of behavioral and physiological parameters for the assessment of postoperative analgesic demand in newborns, infants, and young children: a comprehensive report on seven consecutive studies. Paediatric Anesthesia, 10, 303-318.

Carbajal, R., Eble, B., \& Anand, K. J. (2007). Premedication for intubation in neonates: Confusion or controversy? Seminars in Perintatology, 31, 309-317.

Choong, K., Alfaleh, K., Doucette, J., Gray, S., Rich, B., Verhey, H., et al. (2010). Remifentanil for endotracheal intubation in neonates: a randomized controlled trial. Archives of Disease in Childhood Fetal and Neonatal Edition, 95, 80-84.

Cook-Sather, S. D., Tulloch, H. V., Cnaan, A., Nicolson, S. C., Cubina, M.L., Gallagher, P.R. et al. (1998). A comparison of awake versus paralyzed tracheal intubation for infants with pyloric stenosis. Anesthesia and Analgesia, 86, 945-951. 
Dempsey, E. M., Al Hazzani, F., Faucher, D., \& Barrington, K. J. (2006). Facilitation of neonatal endotracheal intubation with mivacurium and Fentanyl in the neonatal intensive care unit. Archives of Diseases in Childhood Fetal and Neonatal Edition, 91, 279-282.

Dodd, M., Janson, S., Facione, N., Faucett, J., Froelicher, E. S., Humphreys, J. et al. (2001). Advancing the science of symptom management. Journal of Advanced Nursing, 33, $668-676$.

Duncan, H. P., Zurick, N. J. \& Wolf, A. R. (2001). Should we reconsider awake neonatal intubation? A review of the evidence and treatment strategies. Pediatric Anesthesia, 11, 135-145.

Ellis, J. A., McCleary, L., Blouin, R., Dube, K., Rowley, B., MacNeil, M., et al. (2007). Implementing best practice pain management in a pediatric hospital. Journal for Specialists in Pediatric Nursing, 12, 264-277.

Evans, J. (2001). Physiology of acute pain in preterm infants. Newborn and Infant Nursing Reviews, 1, 75-85.

Ferrell, B. \& McCaffery, M. Knowledge and attitudes survey regarding pain. Retrieved August 13, 2008 from http://prc.coh.org

Franck, L., Greenberg, C., \& Stevens, B. (2000). Pain assessment in infants and children. Pediatric Clinics of North America, 47, 487-511.

Ghanta, S., Abdel-Latif, M. E., Lui, K., Ravindranathan, H., Awad, J., \& Oei., J. (2006). Propofol compared with the morphine, atropine, and suxamethonium regimen as induction agents for neonatal endotracheal intubation: a randomized controlled trial. Pediatrics, 119, 1248-1255. 
Grunau, R., Weinberg, J., \& Whitfield, M. F. (2004). Neonatal procedural pain and preterm cortisol response to novelty at 8 months [Electronic version]. Pediatrics, 114, e77-e84.

Grunau, R. E., Holsti, L., Haley, D. W., Oberlander, T., Weinberg, J., Solimano, A., et al. (2005). Neonatal procedural pain exposure predicts lower cortisol and behavioral reactivity in preterm infants in the NICU. Pain, 113, 293-300.

Hassid, S., Nicaise, C., Michel, F., Vialet, R., Thomachot, L., Lagier, P., \& Martin, C. (2007). randomized controlled trial of sevoflurane for intubation in neonates. Pediatric Anesthesia, 17, 1053-1058.

International Association for the Study of Pain. (2010). Pain definitions. Retrieved November 28, 2010 from http://www.iasp-pain.org/

Kanwaljeet, J.S., Aranda, J. V., Berde, C. B., Buckman, S., Capparelli, E. V., Waldemar, C. et al. (2006). Summary proceeding from the neonatal pain-control group. Pediatrics, 117, S9-S22.

Kelly, M. A. \& Finer, N. N. (1984). Nasotracheal intubation in the neonate: Physiologic responses and effects of atropine and pancuronium. Journal of Pediatrics, 105, 303-309.

Kumar, P., Denson, S., Mancuso, T., and Committee on Fetus and Newborn, Section on Anesthesiology and Pain Medicine. (2010). Premedication for nonemergency intubation in the neonate. Pediatrics, 125, 608-615.

Lawrence, J., Alcock, D., McGrath, P., Kay, J., MacMurray, S. B., \& Dulberg, C. (1993). The development of a tool to assess neonatal pain. Neonatal Network, 12, 59-66. 
Lemyre, B., Doucette, J., Kalyn, A., Gray, S., \& Marrin, M. L. (2004). Morphine for elective endotracheal intubation in neonates: a randomized trial. BMC Pediatrics, 4, $232-238$.

Lidow, M. (2002). Long-term effects of neonatal pain on nociceptive systems. Pain, 99, $377-383$.

McKechnie, L., \& Levene, M. (2007). Procedural pain guidelines for the newborn in the United Kingdom. Journal of Perinatology, 27, S1-S5.

McMillian, D.D., Rademaker, A.W., Buchan, K.A., Reid, A., Machin, G., \& Sauve, R.S. (1986). Benefits of orotracheal and nasotracheal intubation in neonates requiring ventilatory assistance. Pediatrics 77, 39-44.

Miller, C. \& Bisonnette, B. (1994). Awake intubation increased intracranial pressure without affecting cerebral blood flow velocity in infants. Canaedian Journal of Anaestheia, 41, 281-287.

Naulaers, G., Deloof, E., Vanhole, C., Kola, E., \& Devlieger. (1997). Use of methohexital for elective intubation in neonates. Archives of Diseases in Childhood Fetal and Neonatal Edition, 77, 61-64.

Oei, J., Butha, T., \& Lui, K. (2002). Facilitation of neonatal nasotracheal intubation with premedication: a randomized controlled trial. Journal of Pediatric Child Health, 38, 146-150.

Peters, J. W., Koot, H. M., \& Grunau, R. E. (2003). Neonatal facial coding system for assessing postoperative pain in infants: item reduction is valid and feasible. Clinical Journal of Pain, 19, 353-363. 
Porter, F.L., Wolf, C., Gold, J., Lotsoff, D., \& Miller, J. P. (1997). Pain and pain management in the newborn in newborn infant: a survey of physicians and nurses. Pediatrics, 100, 626-632.

Roberts, K. D., Leone, T. A., Edwards, W. H., Rich, W. D., \& Finer, N. N. (2006). Premedication for nonemergent neonatal intubations: a randomized controlled trial comparing atropine and Fentanyl to atropine, Fentanyl, and mivacurium. Pediatrics, $118,1583-1591$.

Sakar, S., Schumacher, R. E., Baumgart, S., \& Donn, S. M. (2006). Are newborns receiving premedication before elective intubation? Journal of Perinatology, 26, 286-289.

Shah, V., \& Ohlsson, A. (2002). The effectiveness of premedication for endotracheal intubation in mechanically ventilated neonates: a systematic review. Clinics in Perinatology, 29, 535-554.

Silva, Y. P., Gomez, F. S., de Oliveira, M. J., Maximo, t. A., Barbosa, R. F., \& Silva, C. S. (2007). Morphine versus remifentanil for intubation preterm neonates. Archives of Diseases in Childhood Fetal and Neonatal Edition, 92, F293-F294.

Simon, L., Trifa, M., Mokhtari, M., Hamza, J., \& Treluyer, J. (2004). Premedication for tracheal intubation: a prospective survey in 75 neonatal and pediatric intensive care units. Critical Care Medicine, 32, 565-568.

Stevens, B. J. \& Johnston, C. C. (1994). Physiologic responses of premature infants to a painful stimuli. Nursing Research, 43, 226-231.

Stevens, B., Johnston, C., \& Petryshen, P. (1996). Premature infant pain profile: development and initial validation. Clinical Journal of Pain, 12, 13-22 
Stevens, B., McGrath, P., Gibbons, S., Beyene, J., Breau, L., Camfield, C., et al. (2003). Procedural pain in newborns at risk for neurologic impairment. Pain, 105, 27-35.

Taddio, A., Katz, J., Ilersich, A. L. \& Koren, G. (1997). Effect of neonatal circumcision on pain response during subsequent routine vaccination. Lancet, 349, 599-603.

University of California, San Francisco School of Nursing Symptoms Management Faculty Group. (1994). A model for symptom management. Image: Journal of Nursing Scholarship, 26, 272-276.

VanLooy, J. W., Schumacher, R. E. \& Bhatt-Mehta, V. (2008). Efficacy of a premedication algorithm for nonemergent intubation in a neonatal intensive care unit. Annals of Pharmacotherapy, 42, 947-955.

Venkatesh, V., Ponnusamu, V., Anandaraj, J, Chaudhary, R., Malviya, M., Clarke, P., et al., (2010). Endotracheal intubation in a neonatal population remains associated with a high risk of adverse events. Retrieved October 14, 2010 from European Journal of Pediatrics.

Walter-Nicolet, E., Flamant, C., Negrea, M., Parat, S., Hubert, P., \& Mitanchez, S. (2007). Premedication before tracheal intubation in french neonatal intensive care units and delivery rooms. Archives of Pediatrics, 14, 144-149.

Warnock, F. \& Sandrin, D. (2004). Comprehensive description of newborn distress behavior in response to acute pain newborn male circumcision. Pain, 107, 242-255.

West Virginia United Health System (2008). Mission statement. Retrieved March 30, 2008 from www.wvunitedhealthsystem.org/mission.html

West Virginia University Health Science Center (2008). Mission statement. Retrieved March 30, 2008 from www.hsc.wvu.edu/? 1 
Whyte, S., Birrell, G., Jonathan, Wyllie, \& Woolf, A. 2000). Premedication before intubation in UK neonatal units. Archives of Diseases in Childhood Fetal and Neonatal Edition, 82, 38-41.

World Health Organization. (2007). Sensation of pain. Retrieved October 14, 2007, from http://www.who.int/classifications/icf/site.

Young, T. E. \& Mangum, B. (Eds.). (2007). Neofax (20 $0^{\text {th }}$ ed.). Raleigh, NC: Acorn. 